TITLE:

\title{
Open field equilibrium current and cross-field passing electrons as an initiator of a closed flux surface in EC-heated toroidal plasmas
}

\section{AUTHOR(S):}

Maekawa, T.; Yoshinaga, T.; Uchida, M.; Watanabe, F.; Tanaka, $\mathrm{H}$.

\section{CITATION:}

Maekawa, T. ... [et al]. Open field equilibrium current and cross-field passing electrons as an initiator of a closed flux surface in EC-heated toroidal plasmas. Nuclear Fusion 2012, 52(8): 83008.

ISSUE DATE:

2012-07-11

URL:

http://hdl.handle.net/2433/160030

\section{RIGHT:}

C 2012 IAEA, Vienna; この論文は出版社版でありません。引用の際には 出版社版をご確認ご利用ください。; This is not the published version. Please cite only the published version. 


\title{
Open field equilibrium current and cross field passing electrons as an initiator of closed flux surface in EC-heated torus
}

\author{
T Maekawa ${ }^{1}$, T Yoshinaga ${ }^{2}$, M. Uchida ${ }^{1}$, F. Watanabe ${ }^{1}$ and H. \\ Tanaka $^{1}$ \\ ${ }^{1}$ Graduate School of Energy Science, Kyoto University, Kyoto 606-8502, Japan \\ ${ }^{2}$ National Defense Academy of Japan, Yokosuka 239-8686, Japan \\ E-mail: maekawa@energy.kyoto-u.ac.jp
}

\begin{abstract}
A modeling for the non-inductive initiation of a closed flux surface observed in electron cyclotron (EC) heated toroidal plasmas is presented. First, a pressure driven equilibrium toroidal current develops under a weak external vertical field so as to counter balance the pressure-ballooning and current-hoop forces. When the self field from the current almost cancels out the external vertical field, a forward energetic part of electrons in the velocity space begin to make cross field passing (CFP) orbits. The CFP electrons are generated by the EC heating of bulk electrons and subsequent pitch angle scattering, which is analyzed using the Fokker Planck equation. They provide an additional current that closes the filed lines. The model is examined for experiments in the small low aspect ratio device of LATE and in the large conventional device of JT-60U with a search for appropriate modes of EC heating. Simultaneous coincidence of the model with these two experiments is obtained in terms of microwave power and driven current. The results predict that initiation of closed flux surface requires more and more EC power as the plasma major radius increases. Especially, careful injection of high $N_{\|}$EC waves are needed for large devices, both for initiation of a closed flux surface and for subsequent enlargement of the flux surface by usual EC current drive (ECCD) onto the closed flux area.
\end{abstract}




\section{Introduction}

A toroidal current was generated in a number of experiments when a weak vertical field, $B_{V}$, was superposed in EC- heated toroidal plasmas $[1,2,3,4,5,6,7,8,9,10,11,12]$. The current increased as EC power was increased, and often, a closed flux surface was initiated via a rapid current increase (current jump) in small low aspect ratio devices $[4,5,7,9,10]$. Once a closed flux surface was formed, even current ramp-up by EC current drive (ECCD) was possible [13]. These experimental results are not only interesting but also important since realization of compact tokamak reactors depends on reduction or removal of a central solenoid from the reactors $[14,15]$. Even in conventional tokamak reactors with a full central solenoid such as ITER [16], non-inductive formation of an initial closed flux surface by EC power is useful to save the flux of central solenoid and to prolong the burning time.

(a)

Helical Field Lines

at $\mathrm{R}=13.5 \mathrm{~cm}$ and $27 \mathrm{~cm}$

Toroidal field :

$\mathrm{B}_{\phi}=480$ Gauss at $\mathrm{R}=25 \mathrm{~cm}$

Vertical field :

$\mathrm{B}_{\mathrm{V}}=120$ Gauss (Uniform)
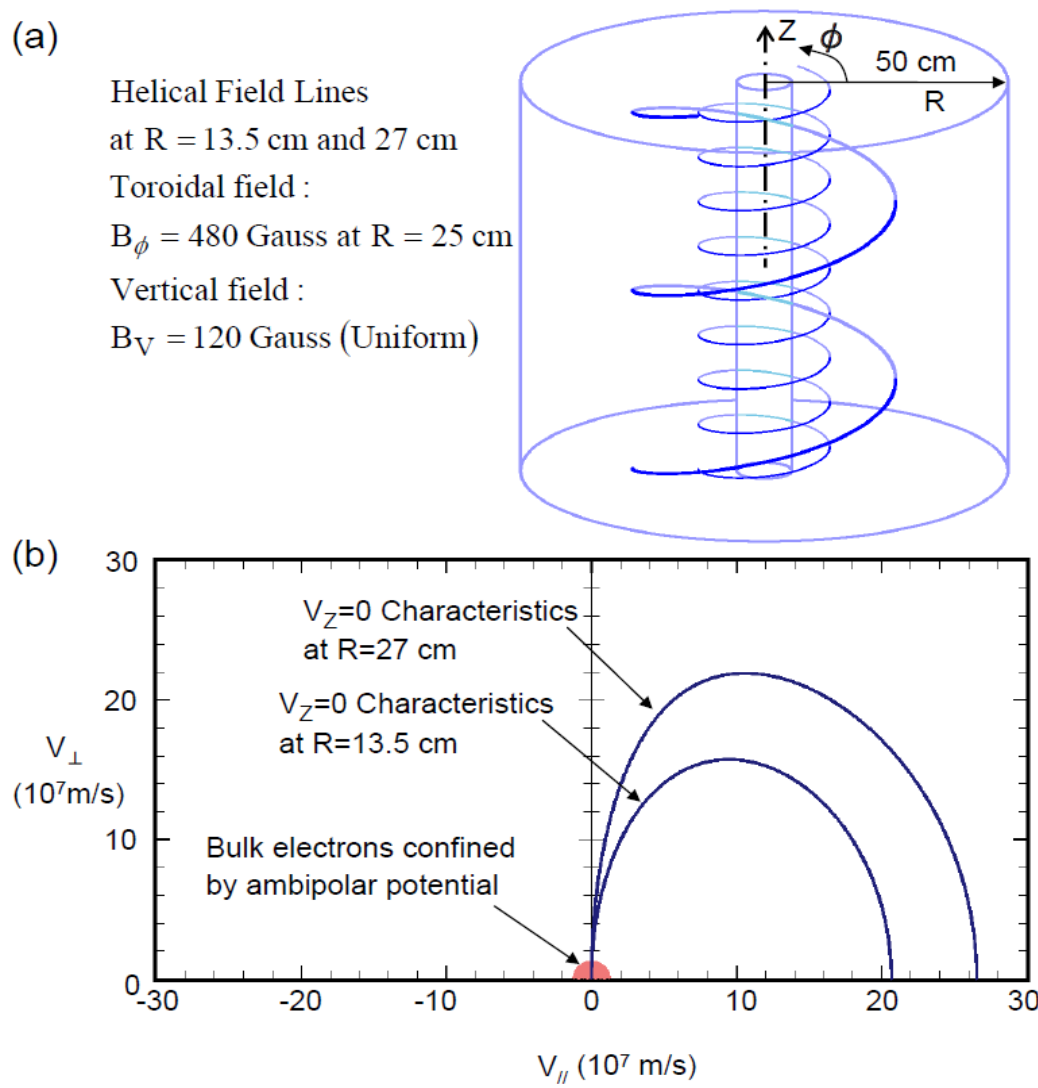

Figure 1. (a) Helical field lines by the superposition of a vertical field $B_{V}$ on the toroidal field $B_{\phi}$ and (b) the $V_{Z}=0$ characteristics in the electron velocity space

In order to understand the current generation in helical external field lines composed of $B_{V}$ and the toroidal filed $B_{\phi}$ (see figure 1 (a)), two mechanisms were proposed. They can be qualitatively described using the guiding-center-drift description for the EC-heated electrons as follows. Suppose an axisymmetric plasma is immersed in a toroidal magnetic field in the cylindrical coordinates of $(R, \phi, Z)$ with $\mathrm{Z}$ coordinate on 
the symmetric axis. Each charged particle drifts vertically across the field lines due to the radial gradient and curvature of $B_{\phi}$ with the velocity given by

$$
V_{V T F}=\frac{m \gamma\left(v_{\|}^{2}+v_{\perp}^{2} / 2\right)}{q B_{\phi} R}
$$

Here $q$ and $m$ is the charge and mass ; $\gamma$ is the relativistic factor and $\|$ and $\perp$ denotes the parallel and perpendicular components of the velocity to the field, respectively. Hereafter, this vertical drift is referred to as vacuum-toroidal-field (VTF) drift since the torodal field in these EC-heated plasmas is essentially vacuum field with negligibly small paramagnetic and diamagnetic effects. We assume $B_{\phi}>0$ and $B_{V}>0$ throughout the paper without loss of generality. Then, the electrons $(q=-e<0$, where e is the elementary charge) VTF-drift downward while the ions $(q>0)$ do upward.

The first mechanism of current generation is based on a preferential confinement of energetic tail electrons in the collisionless energy range. In the presence of $B_{V}$ the vertical drift velocity of the collisionless electron is a sum of the VTF drift velocity and the vertical component of the parallel drift velocity along the helical field line and is, for a uniform $B_{V}$ field, approximately given by

$$
V_{Z}=v_{\|} \frac{B_{V}}{B_{\phi}}-\frac{m_{e} \gamma\left(v_{\|}^{2}+v_{\perp}^{2} / 2\right)}{e B_{\phi} R}
$$

When $V_{Z}=0$, the electron makes a circular orbit along the toroidal field line and is confined. These $V_{Z}=0$ orbits realize only when the parallel drift velocity is positive and the first term exactly compensates the negative VTF drift. On the other hand electrons with negative parallel velocity have negative $V_{Z}$ and are lost downward to the bottom of the vessel, manifesting asymmetric confinement between the forward and backward drift along the field line, which can generate a toroidal current [17]. The $V_{Z}=0$ condition makes an ellipsis-like characteristics in the velocity space as shown in figure 1(b). Here, the current due to this preferential confinement for collisionless tail electrons is temporarily referred to as $j_{T P C}$. The asymmetric confinement area in the electron velocity space more or less expands from the initial narrow area of the $V_{Z}=0$ characteristics when the self poloidal field from $j_{T P C}$ is took into account. Then, the expansion in confinement area would increase the current further. This chain of improvement, that is, a positive feedback was demonstrated using a zero dimensional Fokkar Plank code [18], which might account for the current jump. However, growth of $j_{T P C}$ via this positive feedback is not the case since it requires an unrealistically huge EC power as inferred from the results in the present paper.

While some electrons around the $V_{Z}=0$ characteristic line become mirror-confined when the $B_{V}$ field is not uniform and has a finite decay index, almost electrons have lost orbits. This situation leads to the production of an ambi-polar potential in the order of $V_{a m b} \sim T_{e} / e[19]$ to balance the electron and ion flows along the filed line to the wall. Thermal electrons are confined by this potential. 
The second mechanism of current generation is based on the VTF drift of thermal electrons. The VTF drift of collisional bulk electrons in the thermal energy range may result in a vertical charge separation current, which is given by summing up the VTFdrift current of every electron over the Maxwell velocity distribution and is written in terms of the local bulk electron pressure $p_{e}$ as;

$$
j_{V T F}=\frac{2 p_{e}}{R B_{\phi}}
$$

Here we neglect ion contribution since ion temperature is much lower than the electron temperature in the EC-heated plasmas in the open fields. This vertical current gives rise a charge separation. In a simple toroidal field without $B_{V}$ the vertical charge separation results in a current that circulates via the conducting vacuum vessel, which was predicted many years ago [20] and experimentally confirmed recently [21, 22]. When a weak vertical field $B_{V}$ is superposed the charge separation may be compensated by the return current along the helical field line around the center post (see figure 1(a)) [23], resulting in a toroidal current, $j_{E Q L}$, which is approximately given by

$$
j_{E Q L}=-j_{V T F} \frac{B_{\phi}}{B_{V}}=-\frac{2 p_{e}}{R B_{V}}
$$

when the self field from $j_{E Q L}$ itself is neglected.

In terms of fluid description this current is an open field equilibrium current to balance the outward pressure ballooning force of bulk electrons $\left(2 p_{e} / R\right)$ by the counter force with the interaction with $B_{V}\left(\mathbf{j}_{E Q L} \times \mathbf{B}_{V}\right)$ as pointed in [24], which showed for the first time above formula for $j_{E Q L}$. Furthermore the authors of [24] developed a fluid model for open field equilibrium and deduced a set of fluid equations that took the self field into account. They considered the situation and argued that closed flux surfaces could not be generated by equilibrium current alone. This result, therefore, suggested that a combination of the equilibrium current and a current generated by a kinetic effect such as $j_{T P C}$ might be responsible for the production of flux surfaces observed in the experiments.

The equilibrium current, $j_{E Q L}$, changes its direction when $B_{V}$ is reversed. On closed flux surfaces this current generation still works if we replace $B_{V}$ by $B_{Z}$, the local vertical field including the self field. There are, however, always a pair of points on every flux surface; one has $B_{Z}>0$ and the other $B_{Z}<0$. Therefore, the local current directions are opposite between the pair locations and net toroidal current is hardly generated on closed flux surfaces. Furthermore preferential confinement for tail electrons essentially disappears and therefore there is no $j_{T P C}$ on closed flux surfaces. Closed flux surfaces may be maintained by the EC waves driven current, $j_{E C C D}$, and/or bootstrap current [3]. In both cases the current carriers are directional passing electrons that circulate around the torus along the field lines on the flux surfaces. Thus the current generation mechanisms are quite different between in open and closed fields. Then a question arises how the field changes from open to closed one. 
A sketch of a scenario that combined $j_{E Q L}$ and $j_{T P C}$ was proposed to explain the initiation of a closed flux surface via a current jump observed in the LATE device as follows [5]. First the open field equilibrium current, $j_{E Q L}$, increases as the bulk electron pressure increases and the self field from this current develops at the inboard side of the current channel, where $B_{Z}$ is reduced significantly from $B_{V}$ by the self field (See $B_{V}$ and $B_{Z}$ profiles in figure 3$)$. Then asymmetric confinement area in the electron velocity space expands drastically from the initial narrow area of the $V_{Z}=0$ ellipsis (the confinement area in vacuum field (figure 1(b)) expands as that in figure 5 (a), and then $5(\mathrm{~b}))$. At the same time tail orbits also change from simple $V_{Z}=0$ orbits in the external field to cross field passing (CFP) orbits. The expansion of confinement area induces the second event. Namely, asymmetrically confined tail electrons generated by EC power, which are referred to as CFP electrons in the present paper, increase drastically; then a fast positive feedback of current generation begins, this time, by the assist from the equilibrium current. This leads to the current jump observed in small devices (see $[4,5])$. Once closed flux surfaces are formed via current jump, ECCD begins to work and maintain the closed flux surface. Thus, the CFP electrons bridge the open field equilibrium maintained by $j_{E Q L}$ to the closed filed one maintained by $j_{E C C D}$.

While this scenario improved the original feedback model that depended solely on the tail current generated by the preferential confinement, there remained important questions and issues. First, whether or not the CFP electron current flows at the inboard side of the equilibrium current where the $B_{Z}$ field is most reduced and, therefore, the CFP electron current can most easily close the flux surface. Second, what is the distribution of EC-heated CFP electrons on the velocity space and how much EC power is needed to maintain such a distribution in a given poloidal field composed of $B_{V}$ and the self field from the equilibrium current. Third, how much equilibrium current is needed to trigger the positive feedback of CFP electron current to produce closed flux surfaces by the realistic EC power. Forth, what is the best mode of ECH to generate enough CFP electrons for closing the flux surface and to have the CFP electron distribution smoothly transformed into the ECCD velocity distribution and then to enlarge the initial closed flux surface. These questions are addressed and resolved in the present paper.

Section 2 analyzes the equilibrium characteristics of EC-heated toroidal plasmas in open fields using the fluid description. Even in open fields, radial and vertical force balances are primal to the parallel force balance along the field line [24]. Forces such as a pressure gradient and a centrifugal force associated with a plasma flow are balanced by the $\mathbf{j} \times \mathbf{B}$ force. Since the centrifugal force is neglected in EC-heated torus as explained in this section there may be a pressure governing equation that gives the pressure profile from the information on $\mathbf{j}$ and $\mathbf{B}$. We develop the pressure governing equation from the momentum balance equations. The equation turns out to be consistent with the guiding-center-drift description. We introduce a model current profile that has 8 fitting parameters and produce pairs of model pressure and current profiles for usage in the following sections. It turns out that while the current peak coincides with the $p_{e}$ peak when the self field can be neglected as shown by equation (2), the current peak shifts 
outward along the major radius from the $p_{e}$ peak as the self field increases. Thus the first question whether or not the CFP electron current flows at the inboard side of the equilibrium current is positively resolved since the CFP electrons may be generated around the peak location of $p_{e}$.

Once the field is specified as described above various orbits of energetic electrons can also be specified. In section 3 we develop a Hamiltonian equation for guiding center orbit of energetic electrons using three constants of motion. We neglect effect of electrostatic field on the energetic electrons. For every stating point on the EC resonance (ECR) layer along which the $p_{e}$ peak runs as a ridge, we can obtain a confinement mapping on the velocity space by tracing out the orbits that start with various velocities and pitch angles.

In section 4 we develop a simplified Fokker Plank model including the diffusion by EC wave to deduce the velocity distribution of energetic electrons on the confinement mapping. Once the velocity distribution is estimated, we can calculate the current distribution carried by energetic electrons and obtain the flux surface as done in the sections 5 and 6 .

After above preparations we examine the model for the experiments in the LATE device $[4,5,6]$ as a typical case in small low aspect ratio devices, and for the JT-60U experiment [12] as a typical case in conventional large tokamaks, in sections 5 and 6 , respectively. Here, the second and third questions are positively resolved, that is, the CFP electron distribution and the power to generate and maintain them turn out to be reasonable and match to the experiments in LATE and JT-60U, respectively. In section 7 we look for appropriate injection angles and modes of EC waves for large devices to generate enough CFP electrons for closing the flux surface and to have the CFP electron distribution smoothly transformed into the ECCD velocity distribution and then to enlarge the initial closed flux surface, and show that injection of high $N_{\|}$ waves is the solution. The results in sections 5-7 predict that the initiation of a closed flux surface requires more and more EC power as the plasma major radius increases. Especially, the results also suggest that careful control of EC heating and current drive is required in large devices to initiate and enlarge the initial closed flux surface.

After some discussions in section 8, the results are summarized in section 9.

\section{Pressure driven equilibrium current in open fields}

The momentum balance equations both for the electron fluid and ion fluid in a steady state read;

$$
\begin{aligned}
& m_{e} n_{e}\left(\mathbf{u}_{e} \cdot \nabla\right) \mathbf{u}_{e}=-e n_{e}\left(\mathbf{E}+\mathbf{u}_{e} \times \mathbf{B}\right)-\nabla p_{e}+\mathbf{R}_{e i} \\
& m_{i} n_{i}\left(\mathbf{u}_{i} \cdot \nabla\right) \mathbf{u}_{i}=e n_{i}\left(\mathbf{E}+\mathbf{u}_{i} \times \mathbf{B}\right)-\nabla p_{i}+\mathbf{R}_{i e}-\frac{m_{i} n_{i} \mathbf{u}_{i}}{\tau_{C X}}
\end{aligned}
$$

where $\left.\mathbf{u}_{i(e)}=<\mathbf{v}_{i(e)}\right\rangle$ is the ion (electron) fluid velocity ( $\mathbf{v}_{i(e)}$ is the proton (electron) velocity and $<>$ denotes the average over the velocity distribution), $\mathbf{R}_{e i}=-\mathbf{R}_{i e}=$ 
$m_{e} n_{e}<\nu_{e i}>\left(\mathbf{u}_{i}-\mathbf{u}_{e}\right)$ describes the collisional momentum transfer between the electrons and ions. We study the case of a hydrogen plasma and include the ion momentum loss via charge exchange since the degree of ionization of toroidal EC-heated plasmas in open fields is not high. The protons charge-exchange mainly with hydrogen atoms since their charge exchange cross section is quite large, $\sigma_{C X} \sim 10^{-18} m^{2}$ and the charge exchange time is given by $\tau_{C X}=u_{i} / n_{H}\left|<\sigma_{C X} v_{i} \mathbf{v}_{i}>\right|$ where $n_{H}$ is the hydrogen density. By combining above two equations we obtain a single fluid momentum balance equation as follow;

$$
\rho(\mathbf{u} \cdot \nabla) \mathbf{u}=\mathbf{j} \times \mathbf{B}-\nabla p-\frac{\rho \mathbf{u}}{\tau_{C X}}
$$

where $\rho=m_{i} n_{i}, \mathbf{u}=\mathbf{u}_{i}, p=p_{e}+p_{i} \cong p_{e}, \mathbf{j}=e n_{e}\left(\mathbf{u}_{i}-\mathbf{u}_{e}\right)$ and charge neutrality is assumed. Hereafter we take $p=p_{e}$ since $p_{i}$ is negligible compared with $p_{e}$ in the EC-heated plasmas. The $R, \phi, Z$ components of the equation are, respectively,

$$
\begin{aligned}
& \rho(\mathbf{u} \cdot \nabla) u_{R}-\frac{\rho u_{\phi}^{2}}{R}=j_{\phi} B_{Z}-j_{Z} B_{\phi}-\frac{\partial p_{e}}{\partial R}-\frac{\rho u_{R}}{\tau_{C X}} \\
& \rho(\mathbf{u} \cdot \nabla) u_{\phi}-\frac{\rho u_{R} u_{\phi}}{R}=j_{Z} B_{R}-j_{R} B_{Z}-\frac{\rho u_{\phi}}{\tau_{C X}} \\
& \rho(\mathbf{u} \cdot \nabla) u_{Z}=j_{R} B_{\phi}-j_{\phi} B_{R}-\frac{\partial p_{e}}{\partial Z}-\frac{\rho u_{Z}}{\tau_{C X}}
\end{aligned}
$$

We look for axisymmetric solutions that fulfill the constraint for the current,

$$
0=\nabla \cdot \mathbf{j}=\frac{1}{R} \frac{\partial\left(R j_{R}\right)}{\partial R}+\frac{\partial j_{Z}}{\partial Z}
$$

Note that while $p_{e}$ is axisymmetric and no pressure gradient along the toroidal direction there arise a pressure gradient and a plasma flow $\mathbf{u} \cdot \mathbf{B} / B$ along each open field line that reaches the vessel wall.

In equations (3), (4) and (6), pressure gradient is the driving term since the microwave power absorbed by electrons appears initially as a pressure. The second term of left side of equation (4) represents the centrifugal force due to the toroidal component of ion flow. Flow velocity can be attained up to the ion sound velocity $u_{\phi} \approx C_{S} \equiv \sqrt{T_{e} / m_{i}}$ and this term $\left(\sim p_{e} / R\right)$ is smaller than the pressure gradient $\left(\sim p_{e} / a\right)$ by the aspect ratio $a / R_{0}$ where $a$ and $R_{0}$ are the minor and major radii of the plasma loop, respectively. Actually, ion flow velocity may be much lower than the ion sound velocity in EC-heated plasmas, in which there are plenty of neutral particles and, therefore, momentum loss due to the charge exchange is large [25] and the flow velocity is suppressed. Thus, the centrifugal force term may be neglected.

The other drift is due to $\mathbf{E} \times \mathbf{B}$ drift. The parallel component along the field line of electron fluid equation may be simplified by neglecting the inertia term and the collision term as observed in an experiment [22]. Then,

$$
\frac{\partial p_{e}}{\partial \ell} \cong-e n_{e} E_{\|}
$$


Here $\ell$ is the length along the field line. Then the ambi-polar potential in the plasma is estimated as $V_{a m b}=-\int E_{\|} d \ell \sim T_{e} / e$ [19]. Then the perpendicular electrostatic filed is estimated as $E_{\perp} \sim T_{e} /$ ae and the $\mathbf{E} \times \mathbf{B}$ drift velocity is $u_{E \times B} \sim T_{e} / a e B_{\phi}$. Thus, magnitude of the convective terms in equations (4) and (6) is estimated to be $m_{i} n_{i} T_{e}^{2} / a^{3} e^{2} B_{\phi}^{2}$. The ratio of this term to the pressure gradient term $\sim\left(m_{i} / m_{e}\right)\left(r_{L e} / a\right)^{2}$ is quite small, where $r_{L e}$ is the Larmor radius of the thermal electron.

In these circumstances we approximate the radial and vertical components of momentum balance equation (equations (4) and (6)) by retaining the leading terms.

$$
\begin{array}{ll}
R \text {-component }: & j_{\phi} B_{Z}-j_{Z} B_{\phi}=\partial p_{e} / \partial R \\
Z \text {-component }: & j_{R} B_{\phi}-j_{\phi} B_{R}=\partial p_{e} / \partial Z
\end{array}
$$

Equations (7), (8) and (9) constitute the basic set of equations to analyze various equilibrium characteristics of the present axisymmetric plasmas in open fields. By substituting the following relationships from equations (8) and (9),

$$
j_{R}=\frac{1}{B_{\phi}}\left(j_{\phi} B_{R}+\frac{\partial p_{e}}{\partial Z}\right) \text { and } j_{Z}=\frac{1}{B_{\phi}}\left(j_{\phi} B_{Z}-\frac{\partial p_{e}}{\partial R}\right)
$$

into equation (7), we have

$$
\begin{aligned}
0 & =\frac{1}{R} \frac{\partial}{\partial R}\left[\frac{R}{B_{\phi}}\left(j_{\phi} B_{R}+\frac{\partial p_{e}}{\partial Z}\right)\right]+\frac{\partial}{\partial Z}\left[\frac{1}{B_{\phi}}\left(j_{\phi} B_{Z}-\frac{\partial p_{e}}{\partial R}\right)\right] \\
& =\frac{1}{B_{\phi}}\left[\frac{1}{R}\left(j_{\phi} B_{R}+\frac{\partial p_{e}}{\partial Z}\right)+\frac{\partial\left(j_{\phi} B_{R}\right)}{\partial R}+\frac{\partial j_{\phi}}{\partial Z} B_{Z}+j_{\phi} \frac{\partial B_{Z}}{\partial Z}\right] \\
& -\frac{1}{B_{\phi^{2}}}\left[\frac{\partial B_{\phi}}{\partial R}\left(j_{\phi} B_{R}+\frac{\partial p_{e}}{\partial Z}\right)+\frac{\partial B_{\phi}}{\partial Z}\left(j_{\phi} B_{Z}-\frac{\partial p_{e}}{\partial R}\right)\right] \\
& =\frac{1}{B_{\phi}}\left[\frac{2}{R}\left(j_{\phi} B_{R}+\frac{\partial p_{e}}{\partial Z}\right)+\frac{\partial\left(j_{\phi} B_{R}\right)}{\partial R}+\frac{\partial\left(j_{\phi} B_{Z}\right)}{\partial Z}\right]
\end{aligned}
$$

Thus the following relationship holds.

$$
0=\frac{2}{R}\left(j_{\phi} B_{R}+\frac{\partial p_{e}}{\partial Z}\right)+\frac{\partial\left(j_{\phi} B_{R}\right)}{\partial R}+\frac{\partial\left(j_{\phi} B_{Z}\right)}{\partial Z}
$$

Note that this equation is the same as equation (4) in [24] except that equation (11) neglects ion flow along the field line. In the present paper we start from two fluid momentum equations for ions and electrons to show explicitly that this ion flow as well as the $\mathbf{E} \times \mathbf{B}$ drift can be neglected.

We rewritten equation (11) into the pressure governing equation as

$$
\frac{\partial p_{e}}{\partial Z}=-j_{\phi} B_{R}-\frac{R}{2}\left[\frac{\partial\left(j_{\phi} B_{R}\right)}{\partial R}+\frac{\partial\left(j_{\phi} B_{Z}\right)}{\partial Z}\right]
$$

When both the external vertical field and toroidal current profile are specified, corresponding pressure profile is obtained as the solution

$$
p_{e}(R, Z)=p_{v}(R)+p_{a}(R, Z)+p_{b}(R, Z)
$$


where $p_{v}$ is a constant of integration along the Z-coordinate and a function of $R$ and

$$
\begin{aligned}
& p_{a}(R, Z)=-\frac{R j_{\phi} B_{Z}}{2} \\
& p_{b}(R, Z)=-\int^{Z}\left[j_{\phi} B_{R}+\frac{R}{2} \frac{\partial\left(j_{\phi} B_{R}\right)}{\partial R}\right] d Z^{\prime}
\end{aligned}
$$

Here $p_{v}$ drives the vertical current that circulates via the vacuum vessel $[21,22,24]$, and $p_{a}$ and $p_{b}$ drive the toroidal current. Hereafter we define $p_{e}$ as $p_{e}=p_{a}+p_{b}$ and do not concern with $p_{v}$ since $p_{v}$ has nothing to do with the toroidal current.

Equations (8) and (9) indicate that the terms including $j_{\phi}$ or $B_{\phi}$ is the same order leading terms as the pressure gradient term, while all terms in equation (5) are secondary since $[\mathbf{j} \times \mathbf{B}]_{\phi}$ is secondary compared with the terms in equations (8) and (9). Once we have information on pressure profile for a given toroidal current profile we can calculate the diamagnetic and paramagnetic currents using equation (10). Then some information on plasma flow may be obtained using equation (5), which is, however, out of the scope of the paper.

At this point it is useful to examine equation (7) using the guiding-center-drift picture for the VTF drift of electrons. The currents may be composed of the vertical VTF drift current and the force-free return current along the field lines. Then equation (7) takes the following form,

$$
0=\nabla \cdot \mathbf{j}=\frac{1}{R} \frac{\partial\left(R j_{R}\right)}{\partial R}+\frac{\partial\left(j_{V T F}+j_{Z}\right)}{\partial Z}
$$

with

$$
j_{V T F}=\frac{2 p_{e}}{R B_{\phi}}, j_{R}=j_{\phi} \frac{B_{R}}{B_{\phi}} \text { and } j_{Z}=j_{\phi} \frac{B_{Z}}{B_{\phi}} .
$$

It is straightforward to reach equation (11) from equation (14) using low beta approximation for $B_{\phi}$. This result shows that $j_{\phi}$ originates from the VTF-drift of bulk electrons.

We employ a model profile for the toroidal current as described in appendix A. The model can handle broadness (or peaking factor) $\alpha$, triangularity $\delta$ and vertical elongation $\kappa$ of profile, and radial shift of current peak $\sigma$. Figures 2 and 3 show the results for a vertically stretched profile in a small low aspect ratio device. Figure 2 shows the case for a very low current density and figure 3 for a high current density. In the present paper we use a uniform vertical field $\mathbf{B}_{V}=B_{V} \hat{Z}$ for simplicity. In the case of very low current $B_{R}=0$ and $p_{e}=p_{a}=-(R / 2) j_{\phi} B_{V}$ as shown in figure 2 . In the high current case the pressure peak shifts inward from the current peak. In this high current case, $B_{R}$ becomes significant and the field lines bulges outward. Therefore, the pressure driven VTF current returns along the outward path of bulged field lines, resulting in the outward shift of current profile from the pressure profile. The radial profile of poloidal flux ( $\Psi \equiv R A_{\phi}$, where $A_{\phi}$ is the toroidal component of the vector 


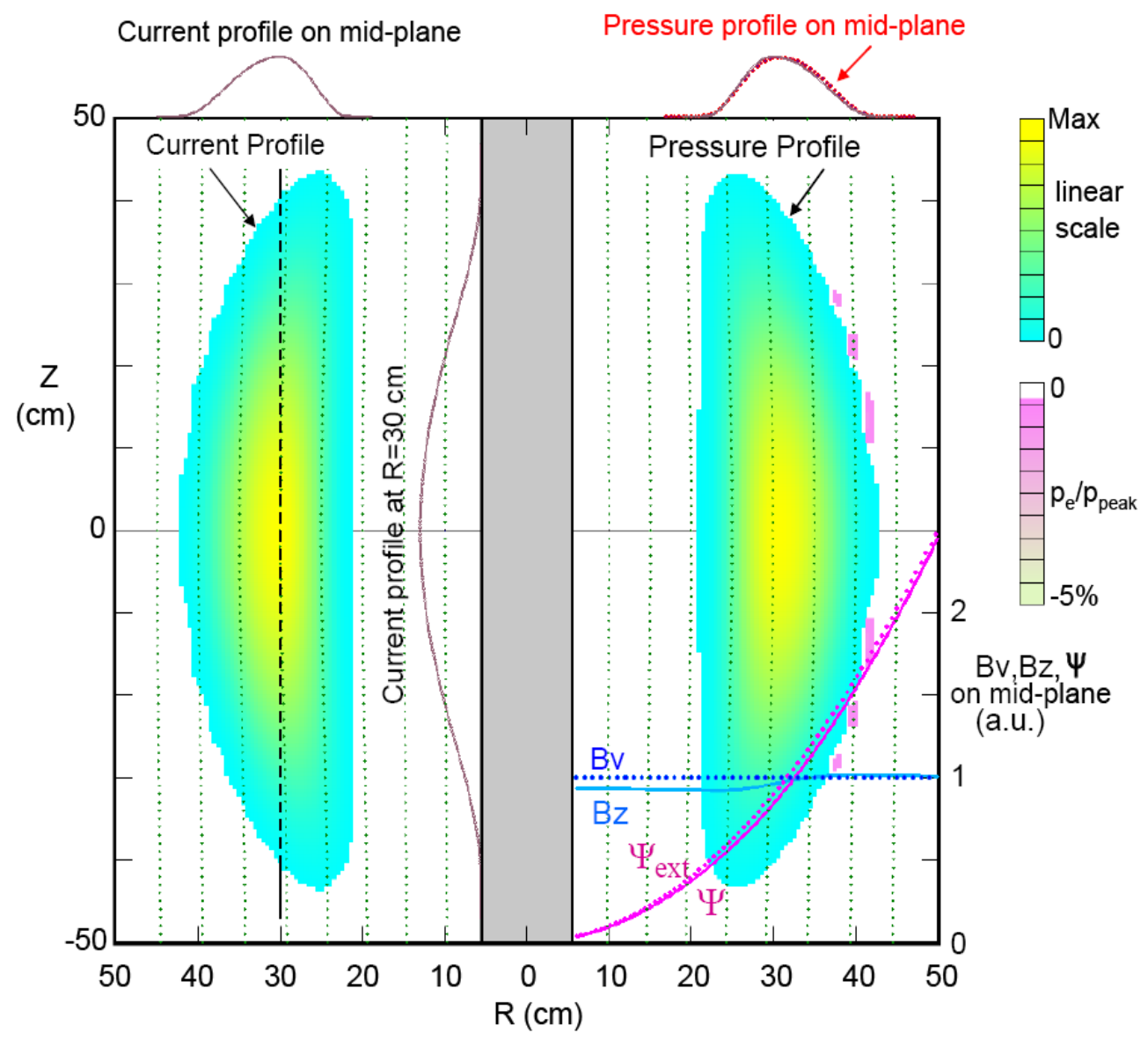

Figure 2. Presure profile in a low current case. $I_{E Q L}=-0.5 \mathrm{kA}, B_{V}=120 \mathrm{G}$, $B_{\phi}=480 \mathrm{G}$ at $R=25 \mathrm{~cm}, j_{\text {peak }}=10.7 \mathrm{kAm}^{-2}$, $\mathrm{p}_{\text {peak }}=19 \mathrm{~Pa}, \alpha=2, \kappa=4.4, \delta=$ $0.7, \sigma=-0.2$, and $\Psi_{e x t}=R^{2} B_{V} / 2$ and $\Psi=\int_{0}^{R} B_{Z} R d R$ with integration on midplane.

potential) on mid-plane is plotted in figures 2 and 3, and also in subsequent similar figures.

While the pressure should be positive, equation (13) does not always give positive pressure profiles for any current profiles. Actually, when the current density is so high that filed lines significantly bulge outward as in figure 3, we need a delicate adjustment of the profile parameters so as to have whole area of pressure profile be positive. Even in the case in figure 3 where the profile has a large triangularity and an inward shift of the peak after intensive adjustment for positive pressure profile, quite weak negative area still remains along the outward boundary and inward boundary. In the case of no triangularity and no shift of peak in the current profile, for example, negative area invades deep into the central part of the profile with increased negativities. These results suggest that the profile in figure 3 have a physical reality matched to strongly EC heated toroidal plasmas in the open fields. Note that in figures 2 and 3 negative area where $p_{e} / p_{\text {peak }}<-0.004$ is also colored and the zero pressure area in the sense 


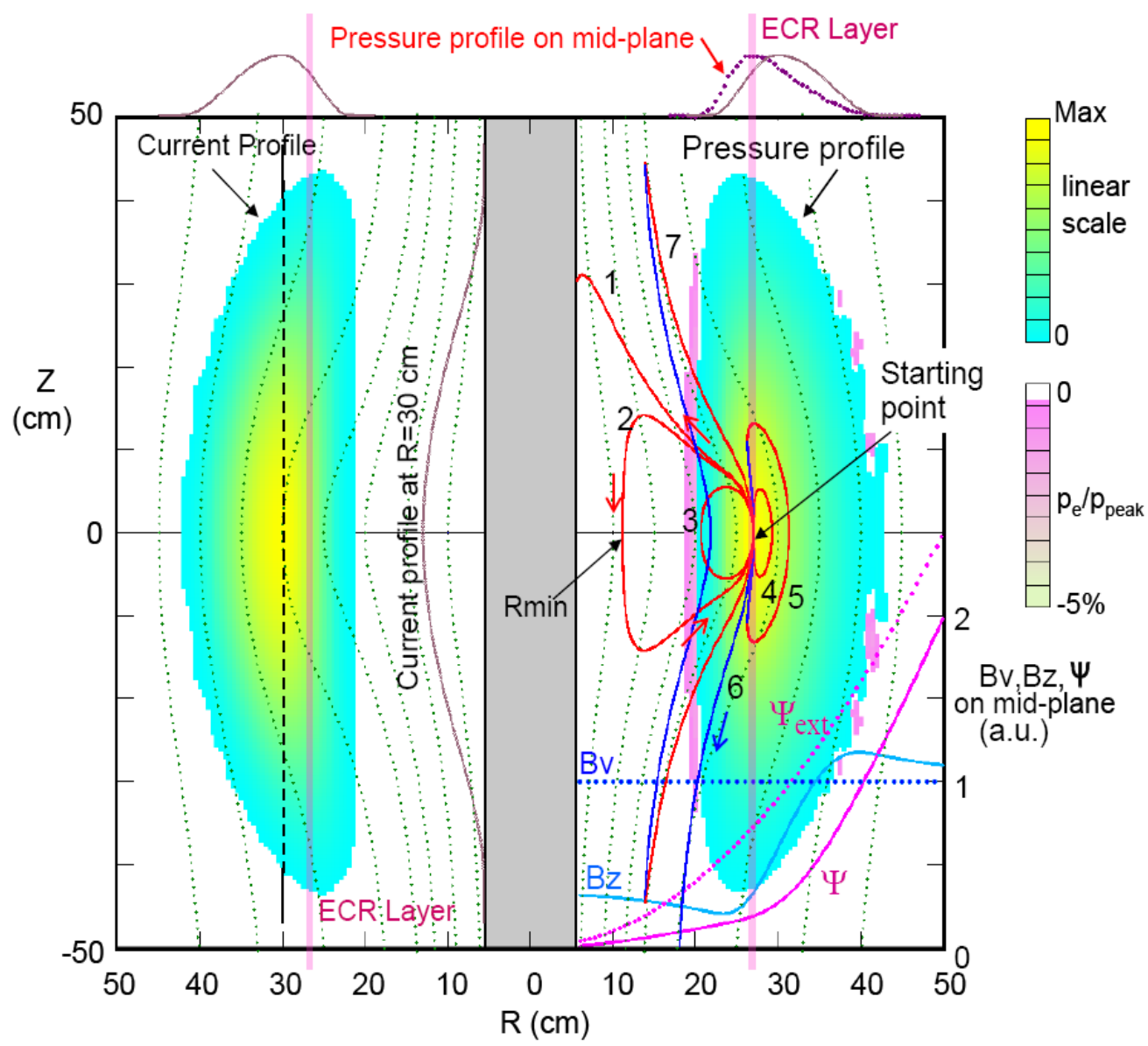

Figure 3. Presure profile in a high current case. $I_{E Q L}=-5.2 \mathrm{kA}, B_{V}=120 \mathrm{G}$, $B_{\phi}=480 \mathrm{G}$ at $R=25 \mathrm{~cm}, j_{\text {peak }}=112 \mathrm{kAm}^{-2}, \mathrm{p}_{\text {peak }}=190 \mathrm{~Pa}, \alpha=2, \kappa=4.4, \delta=$ $0.7, \sigma=-0.2$, and $\Psi_{e x t}=R^{2} B_{V} / 2$ and $\Psi=\int_{0}^{R} B_{Z} R d R$ with integration on midplane. Orbits denoted by 1-7 are guiding center orbits for electrons 1-7 in figure 5(b). Blue denotes the backward drift.

that $-0.004<p_{e} / p_{\text {peak }}<0.004$ remains white.

The profiles fulfill the perpendicular force balance equations (8) and (9). Then they may fulfill the following generalized Shafranov formula with an appropriate $G$ value that reflects geometrical characteristics of the current profile :

$$
B_{V}=\frac{\mu_{0} I_{P}}{4 \pi R}\left[G\left(\frac{R}{a}, \kappa, \ell_{i}, \text { etc }\right)+\beta_{p}\right], \quad \beta_{p}=\frac{8 \pi S<p>}{\mu_{0} I_{P}^{2}} .
$$

Here, $\mathrm{S}$ is the cross section of plasma and $\langle p\rangle$ denotes the average of pressure over the cross section. In the original Shafranov formula for large aspect ratio tori having circular cross sections [26], $G=\ln (8 R / a)+\ell_{i} / 2-3 / 2$. In the present case of low aspect ratio and strongly elongated cross section the profiles indeed fulfill the generalized formula as shown in figure 4, where the $\mathrm{G}$ value is consistent with the estimation by [27]. The figure shows that current-hoop force begins to take part in the radial force balance as $I_{P}$ increases. 


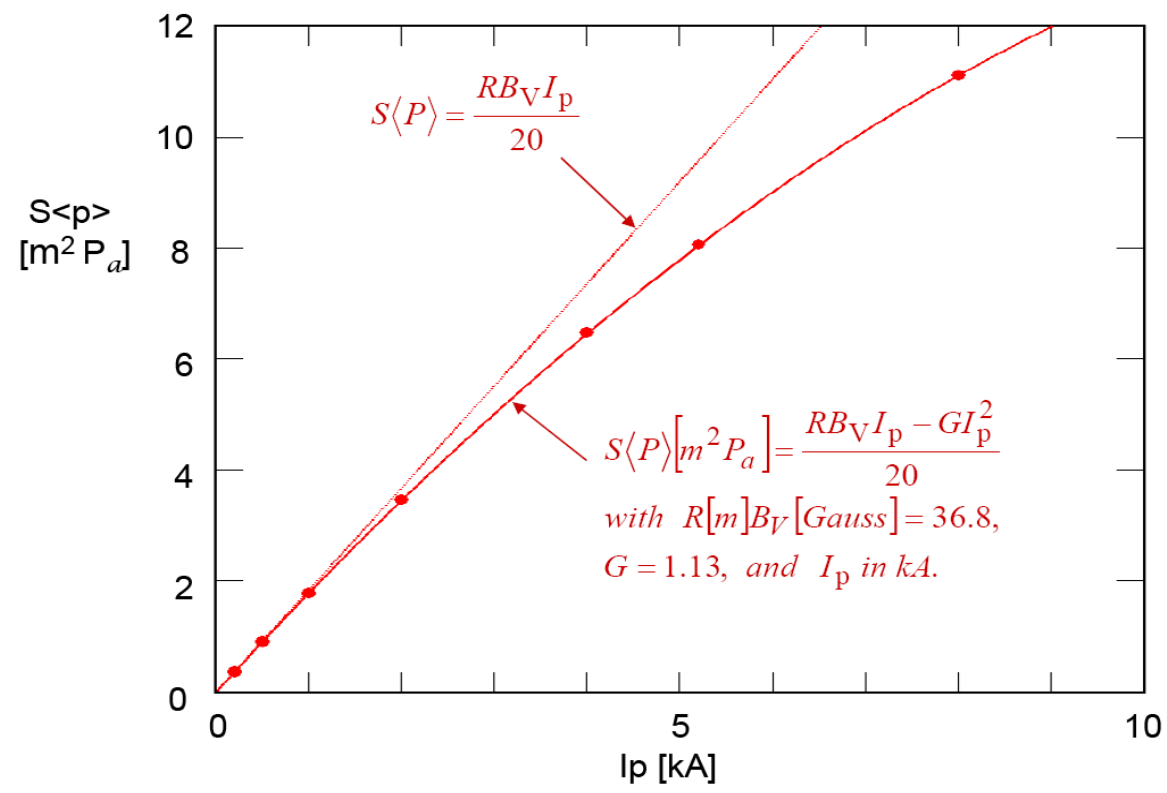

Figure 4. Open field equilibrium fulfills generalized Shafranov formula. The points at $I_{P}=\left|I_{E Q L}\right|=0.5$ and $5.2 \mathrm{kA}$ are those in figures 1 and 2 , respectively.

\section{CFP orbits and confinement mapping}

We are interested in the profile of toroidal current carried by CFP electrons since this current is the most promising candidate that closes the field lines. The electrons are energetic and almost collision-less. Therefore, their guiding-center orbits in the magnetic fields are sufficient to obtain the current profile. Effects from the equilibrium electrostatic electric field are neglected. By taking advantage of the merit that there are three constants of motion in the present case, the guiding-center orbits may be obtained as follows [29]. The constants are the angular momentum, magnitude of the velocity and the magnetic moment as follows,

$$
\begin{aligned}
& P_{\phi} \equiv m R v_{\phi}-e \Psi \cong m R v_{\|}-e \Psi \\
& W \equiv v_{\|}^{2}+v_{\perp}^{2} \\
& \mu \equiv \frac{m v_{\perp}^{2}}{2 B} \cong \frac{m v_{\perp}^{2}}{2 B_{\phi}}
\end{aligned}
$$

where $m=m_{e} \gamma$ and $\gamma$ is the relativistic facor. Then,

$$
W=\frac{2 \mu B_{\phi}}{m}+\left(\frac{P_{\phi}+e \Psi}{m R}\right)^{2}
$$

Here, $B_{\phi}$ is a function of $R$ in the low beta plasmas and the poloidal flux $\Psi=R A_{\phi}$ is a function of $R$ and $Z$. Therefore $W$ is a function of $R$ and $Z$ for a set of the constants $P_{\phi}$ and $\mu$. 


$$
W=W\left(P_{\phi}, \mu ; R, Z\right)
$$

We calculate the trajectory of electron projected on the poloidal cross section. The coordinates $R$ and $Z$ are functions of the distance $\ell$ along the trajectory from the starting point. Then the following deferential equations describe the evolutions of $R$ and $Z$.

$$
\frac{d R}{d \ell}=-\frac{\frac{\partial W}{\partial Z}}{\sqrt{\left(\frac{\partial W}{\partial R}\right)^{2}+\left(\frac{\partial W}{\partial Z}\right)^{2}}}, \quad \frac{d Z}{d \ell}=\frac{\frac{\partial W}{\partial R}}{\sqrt{\left(\frac{\partial W}{\partial R}\right)^{2}+\left(\frac{\partial W}{\partial Z}\right)^{2}}}
$$

Azimuthal drift velocity of the guiding center at the location $\mathrm{R}$ and $\mathrm{Z}$ is given by

$$
V_{\phi} \equiv R \frac{d \phi}{d t} \cong v_{\|} \cong \frac{P_{\phi}+e \Psi}{m R}
$$

Radial and vertical drift velocities of guiding center are given by usual way

$$
\begin{aligned}
& V_{R} \equiv \frac{d R}{d t}=v_{\|} \frac{B_{R}}{B_{\phi}} \\
& V_{Z} \equiv \frac{d Z}{d t}=v_{\|} \frac{B_{Z}}{B_{\phi}}-\frac{m\left(v_{\|}^{2}+v_{\perp}^{2} / 2\right)}{e R B_{\phi}}
\end{aligned}
$$

Then

$$
\frac{d \ell}{d t} \equiv \sqrt{\left(\frac{d R}{d t}\right)^{2}+\left(\frac{d Z}{d t}\right)^{2}}
$$

gives the time elapsed along the trajectory and

$$
\frac{d \phi}{d \ell}=\frac{d t}{d \ell} \frac{d \phi}{d t}
$$

gives the toroidal angle of trajectory as a function of $\ell$. Coincidence of the guiding center trajectories with the full trajectories by the full set of equations of motion has been found to be excellent. Typical guiding center orbits starting with various pitch angles and velocities at the starting position of $R_{i}=27 \mathrm{~cm}$ and $Z_{i}=0 \mathrm{~cm}$ (denoted by 1-7 in figure 5(b)) are also plotted in figure 3. The orbits 2,3 and 4 make CFP orbits, respectively.

Full drawing for the orbit 2 including the Larmor motion is shown in figure 6, which most vividly represents characters of the CFP orbit. The electron initially climbs upward since the first term of the right hand side of equation (23) is larger than the second VTF drift term. The electron gradually shifts inward due to the radial component of velocity given by equation (22). As the location shifts inward toroidal field increases as $1 / R$. Then $v_{\|}$decreases so as to conserve magnetic moment and energy (equations (17) and (18)). Due to these changes $V_{Z}$ changes its sign and the electron drifts downward. As 


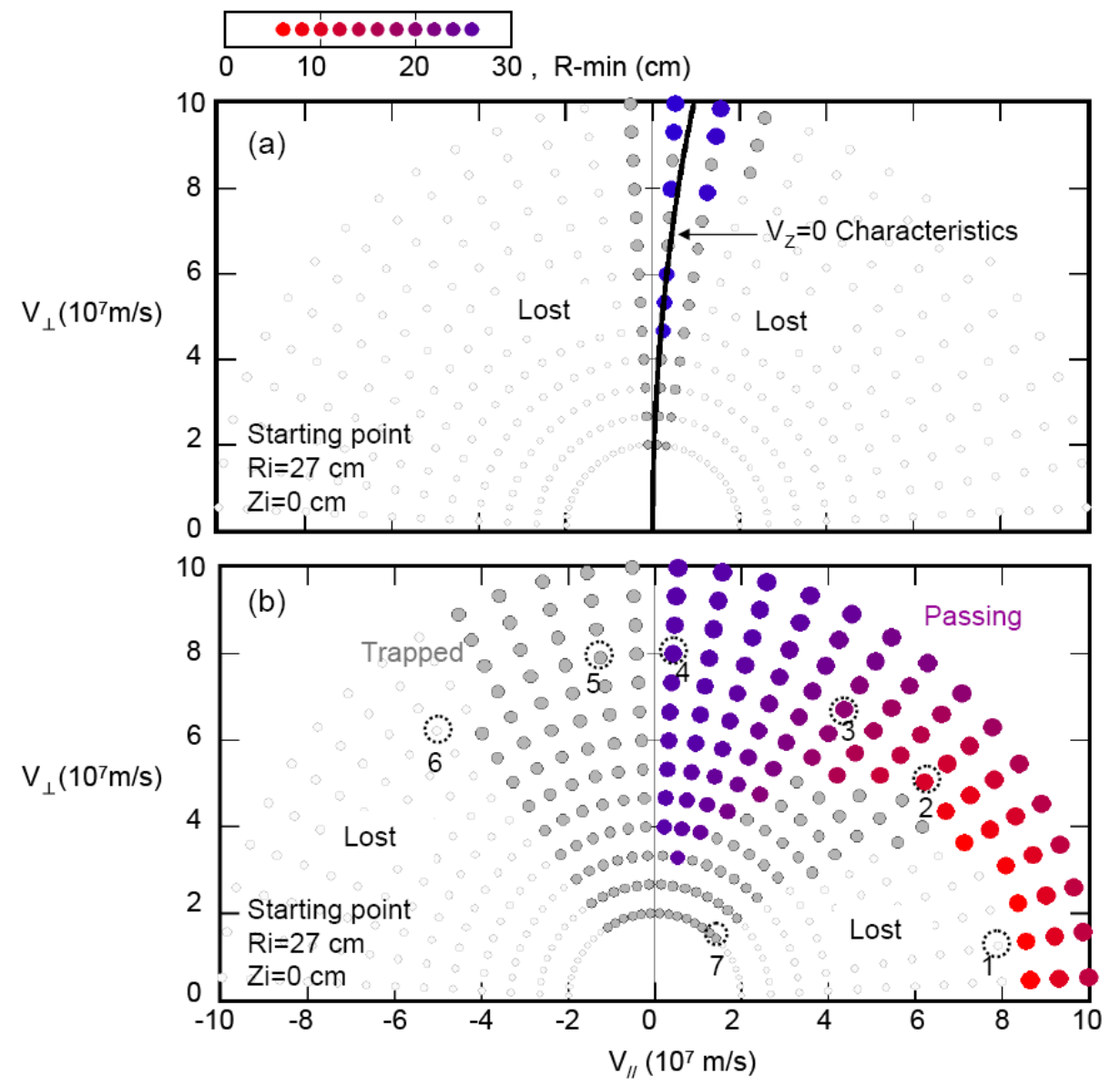

Figure 5. Confinement mapping for electrons start with various pitch angles and velocities. (a): the case in figure 2 and (b): the case in figure 3. Most inward radial locations for passing electron orbits are shown by colors from blue to red.

the electron descends and crosses the mid-plane, $B_{R}$ changes sign and its magnitude increases as the electron further descends. Then the electron moves outward to the lower $B_{\phi}$ side and $V_{Z}$ becomes again positive. Then the electron goes upward, and finally reaches the starting coordinates of $R_{i}$ and $Z_{i}$, closing the orbit in the poloidal section. The outside CFP orbit 4 shown in figure 6 also makes a closed orbit. The characters are the same as the orbit 2 , although they are not so clearly seen as the case 2 .

When the pressure driven equilibrium current is low as the case in figure 2, the vertical field is essentially external one. In this case only electrons in a narrow band around the $V_{Z}=0$ characteristics have confined orbits as shown in figure 5(a). Note that for the range of $v_{\|}<<c$ the $V_{Z}=0$ characteristics is approximately given by

$$
v_{\|} \cong \frac{m v_{\perp}^{2}}{2 e R B_{V}}
$$

When the equilibrium current density increases so high that $B_{Z}$ is significantly lowered at the inboard area of the current channel, the confinement area expands 

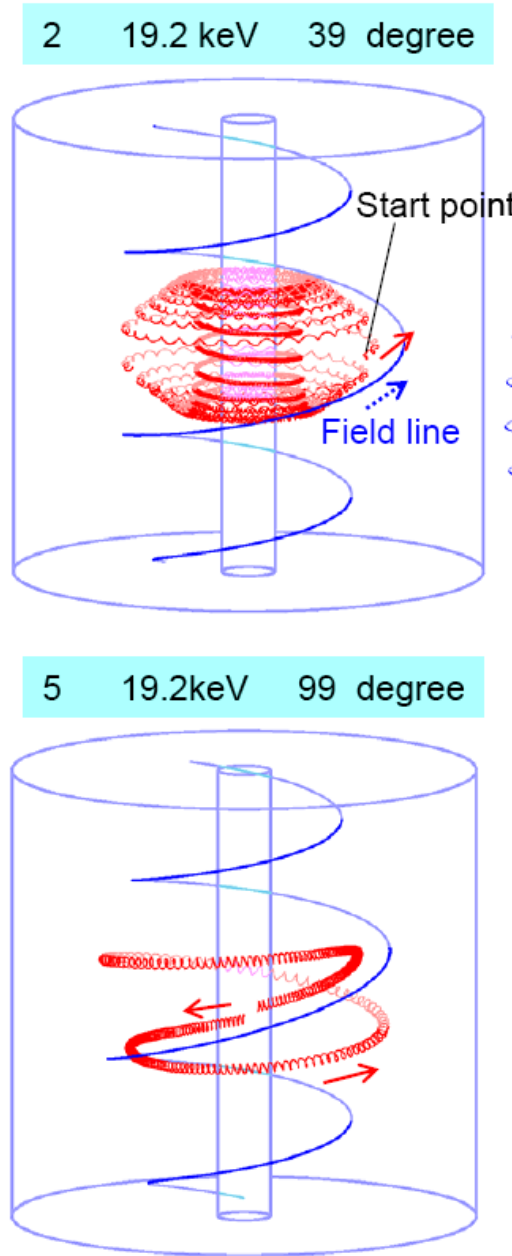
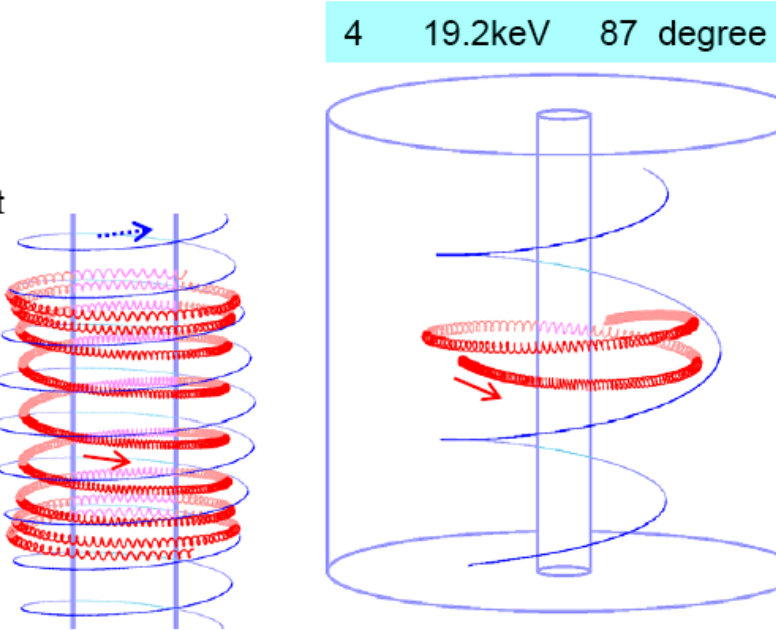

Expanded presentation of the inner $\mathrm{R}$ orbit of 2 with an inner helical field line
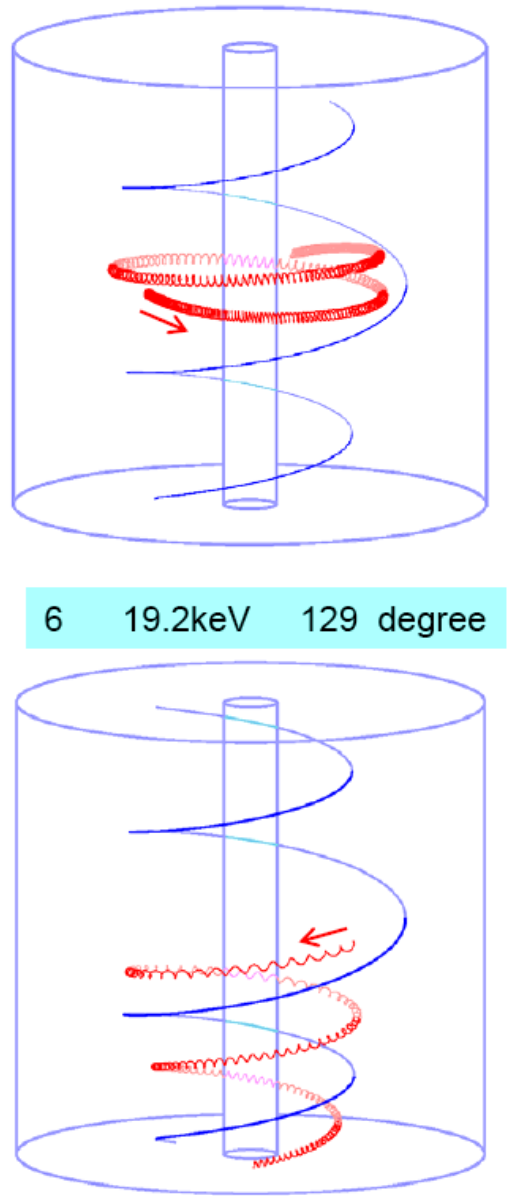

Figure 6. Full orbits for 2, 4, 5 and 6 in figures 3 and 5(b). 2 is an inside passing orbit, 4 is an outside passing orbit , 5 is a trapped orbit and 6 is a lost orbit. Red arrows denote the directions of electron drift.

as shown in Figure 5 (b) and a wide area of electrons around the original $V_{Z}=0$ characteristics in the velocity space begin to make the CFP orbits.

\section{Velocity distribution of EC-heated CFP electrons}

Here we develop a method to obtain the particle distribution of energetic electrons on the confinement mapping in velocity space such as those in figure 5(b). Then we can estimate the additional current carried by CFP electrons on the pressure driven equilibrium current by tracing out every orbit starting with various velocities and pitch angles on the confinement mapping. We begin by assuming that the energetic electrons obey the Fokker-Plank equation [28].

$$
\frac{\partial f}{\partial t}+\nabla \cdot \mathbf{S}_{C}+\nabla \cdot \mathbf{S}_{W}=0
$$


Here $\mathbf{S}_{C}$ and $\mathbf{S}_{W}$ are the fluxes due to collision and wave, respectively. In spherical coordinates with the axisymmetric axis along the direction of magnetic field and the polar angle from the axis $(\theta)$,

$$
\nabla \cdot \mathbf{S}_{C}=\frac{1}{v^{2}} \frac{\partial}{\partial v} v^{2} S_{v}+\frac{1}{v \sin \theta} \frac{\partial}{\partial \theta} \sin \theta S_{\theta}
$$

where

$$
\begin{aligned}
& S_{v}=-\frac{\Gamma}{v^{2}} f, \quad \Gamma=\frac{n_{e} e^{4} \log \Lambda}{4 \pi \epsilon_{0}^{2} m_{e}^{2}} \\
& S_{\theta}=-D_{\theta \theta} \frac{\partial f}{v \partial \theta}, \quad D_{\theta \theta}=\Gamma \frac{1+Z_{e f f}}{2 v}
\end{aligned}
$$

are assumed by retaining the leading terms in the high-velocity limit approximation [28] and $Z_{e f f}=2$ is assumed throughout the paper. $S_{v}$ represents the slowing down flux by collision with the bulk electrons and $S_{\theta}$ does the pitch-angle scattering with the bulk electrons and ions. The flux by EC waves is written by

$$
\nabla \cdot \mathbf{S}_{W}=\frac{1}{v^{2}} \frac{\partial}{\partial v}\left(-v^{2} D_{W} \frac{\partial f}{\partial v}\right)
$$

Here we assume purely perpendicular diffusion along the $\theta=\pi / 2$ line with

$$
D_{W}=\Delta_{W}(v) \delta(v(\theta-\pi / 2)),
$$

where $\Delta \mathrm{w}$ represents the kick in electron energy in passing through the EC resonance.

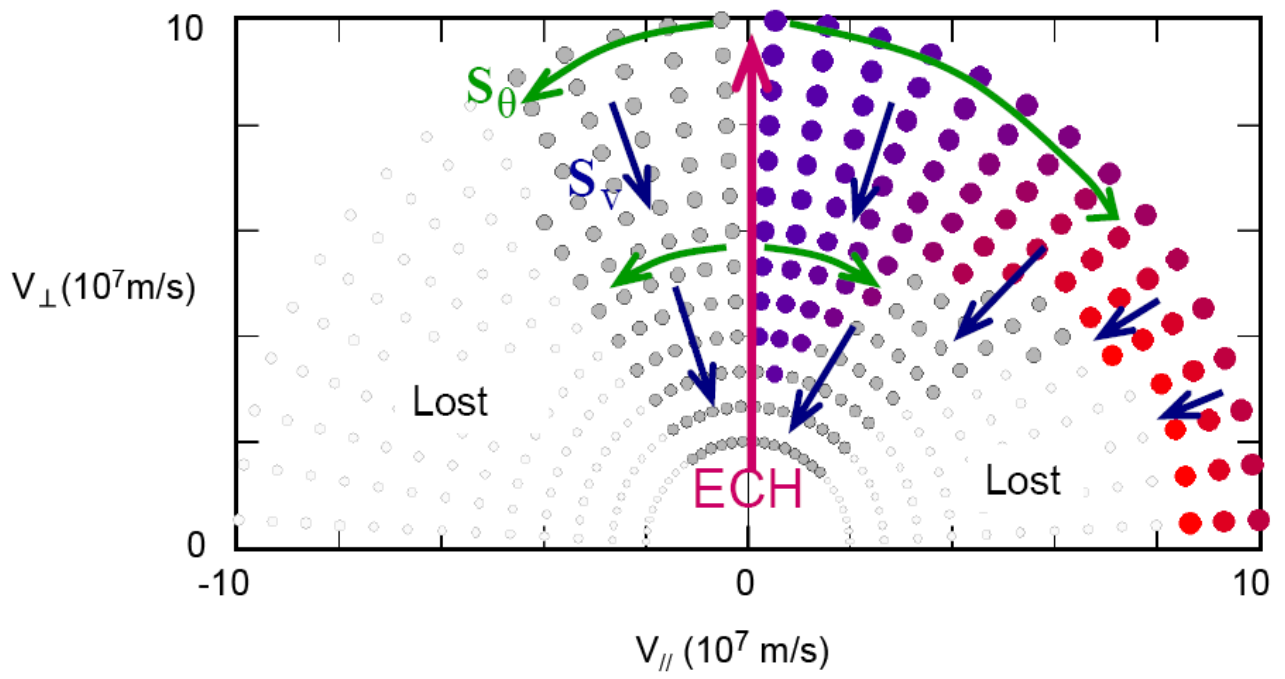

Figure 7. Electron flow in velocity space. $S_{\theta}$ and $S_{v}$ represent pitch angle diffusion and slowing down of energetic electrons by collisions with bulk particles

The above Fokker-Planck model represents the following flow of electrons in the velocity space (see figure 7). First, EC waves pump up electrons perpendicularly into the high energy range from the bulk region. Then the electrons are pitch-angle scattered into forward side as well as backward side. The electrons further diffuse in pitch-angle 
as well as slow-down in velocity and finally are lost when they have reached the lost region.

Indeed above EC-wave-driven diffusion where the direction of diffusion is strictly along the EC resonance line of $\theta=\pi / 2$ is an approximation. First, the EC resonance line is not quite straight along the perpendicular direction as described in Section 7. Second, only when the parallel refractive index $N_{\|} \equiv k_{\|} c / \omega$ is unity such a strict coincidence of diffusion direction and EC resonance line is realized. Actually when $N_{\|} \approx 1$ the resonance line is almost perpendicular and coincidence of both directions is approximately obtained as shown in figure 19. In this case $\Delta_{W}(v)$ hardly depend on $v$ and we can obtain explicit formula for $\Delta_{W}$ using quasi-linear diffusion tensor for a single frequency wave for the present case of the perpendicular diffusion [28] as

$$
\Delta_{W} \cong \frac{\pi}{4} \frac{e^{2}}{m_{e}^{2}} \frac{E_{r} E_{r}^{*}}{k_{\|}},
$$

where $k_{\|}$is the parallel component of the wave number and $E_{r}$ is the right-handed component of the wave electric field and the asterisk denotes the complex conjugate. When $N_{\|}$is much smaller than unity, coincidence between the diffusion direction and the resonance line is obtained only for the lower velocity region and the diffusion direction quickly deviates with $v$ from the resonance line (see the $N_{\|}=0.17$ case in figure 19). In such a case $\Delta_{W}(v)$ would quickly decrease with $v$ from above $\Delta_{W}$ value.

First we look for steady distributions at the outside of pump-up line at $\theta=\pi / 2$. Such velocity distributions obey $\nabla \cdot \mathbf{S}_{C}=0$, that is,

$$
\frac{\partial^{2} f}{\partial \theta^{2}}+\frac{1}{\tan \theta} \frac{\partial f}{\partial \theta}+\frac{2 v}{1+Z_{e f f}} \frac{\partial f}{\partial v}=0
$$

This form has separation of variables solutions $f(v, \theta)=g(v) h(\theta)$ and the differential equation is reduced to

$$
\frac{1}{h}\left(\frac{d^{2} h}{d \theta^{2}}+\frac{1}{\tan \theta} \frac{d h}{d \theta}\right)=-\frac{2 v}{1+Z_{e f f}} \frac{1}{g} \frac{d g}{d v}=C
$$

Then, the solutions take the following form,

$$
g=A v^{-Q}, \quad Q=\frac{C\left(1+Z_{e f f}\right)}{2},
$$

where $A$ and $C$ are constants. The pitch-angle distribution obeys

$$
\frac{d^{2} h}{d \theta^{2}}+\frac{1}{\tan \theta} \frac{d h}{d \theta}=C h
$$

This equation is rewritten as the system of two deferential equations with order 1 for two functions $h(\theta)$ and $s(\theta)$ as follows,

$$
\begin{aligned}
& \frac{d h}{d \theta}=s \\
& \frac{d s}{d \theta}=-\frac{s}{\tan \theta}+C h
\end{aligned}
$$


The solution which meets the boundary conditions for $h(\theta)$ and $s(\theta)$ at each $v$ is numerically obtained and $h$ is normalized by the value at $\theta=\pi / 2$. Then $h$ decreases monotonically from 1 at $\theta=\pi / 2$ towards $\theta=\pi$ or 0 . When the electrons are confined up to the end of $\theta=0$ and $\pi, d h / d \theta=0$ and $h=$ finite at the end. When this is not the case, the electrons are confined up to $\theta_{1}$ and/or $\theta_{2}$, the boundary of the lost area, where $0<\theta_{1}<\pi / 2<\theta_{2}<\pi$. In this case $d h / d \theta>0$ with $h=0$ at $\theta_{1}$ and/or $d h / d \theta<0$ with $h=0$ at $\theta_{2}$.

The flow balance at $\theta=\pi / 2$ requires

$$
2 \pi v d v\left(S_{\theta-}+S_{\theta+}\right)=\frac{1}{v} \frac{\partial}{\partial v}\left(v \Delta_{W} \frac{\partial f}{\partial v}\right) 2 \pi v d v
$$

where

$$
S_{\theta \pm}=\left.\mp \Gamma \frac{1+Z_{e f f}}{2 v} \frac{\partial f}{\partial \theta}\right|_{\theta=\frac{\pi}{2} \pm}
$$

Then we obtain the relationship between $Q$ and $\Delta_{W}$ as follows,

$$
Q=\frac{C\left(1+Z_{e f f}\right)}{2}=\sqrt{\frac{\Gamma\left(1+Z_{e f f}\right)}{2 \Delta_{W} h}\left(\left.\frac{d h}{d \theta}\right|_{\theta=\frac{\pi}{2}-}-\left.\frac{d h}{d \theta}\right|_{\theta=\frac{\pi}{2}+}\right)}
$$

The particle and energy fluxes that are pumped-up into the energetic range are given by

$$
\begin{aligned}
& \text { Particle flux per unit volume }=\int_{v_{0}}^{v_{c}}\left(S_{\theta-}+S_{\theta+}\right) 2 \pi v d v, \\
& \text { Power flux per unit volume }=\int_{v_{0}}^{v_{c}} \frac{m_{e} v^{2}}{2}\left(S_{\theta-}+S_{\theta+}\right) 2 \pi v d v,
\end{aligned}
$$

respectively, where $v_{0}$ and $v_{c}$ are the lower and upper boundary of integration, respectively.

\section{Case in a small low aspect ratio torus}

We examine the model described in sections 2-4 for an experiment in the LATE device described in ref.[6] (see figure 2 in [6]). Modeled open field equilibrium is shown in figure 8. Since EC heating zone is a vertical thin belt along the ECR layer, we adopt 11 sample heating points $\left(Z_{i}=-20,-16,-12,-8,-4,0,4,8,12,16\right.$ and $20 \mathrm{~cm}$ at $\left.R_{i}=19 \mathrm{~cm}\right)$ as the starting points of orbits as partly shown in figure 9 , and add each contribution to obtain the CFP current profile. Here we use the term, CFP current, for simplicity since the contribution from the precession of trapped electrons is negligible compared with that from the CFP electrons. Detailed procedure to obtain the CFP current profile is explained later. The result is shown in figure 10, where magnitude of $B_{Z}$ is lowered almost to zero due to the additional CFP current. Next, we adopt the same sample heating points as the starting points of orbits and calculate the second step CFP current 


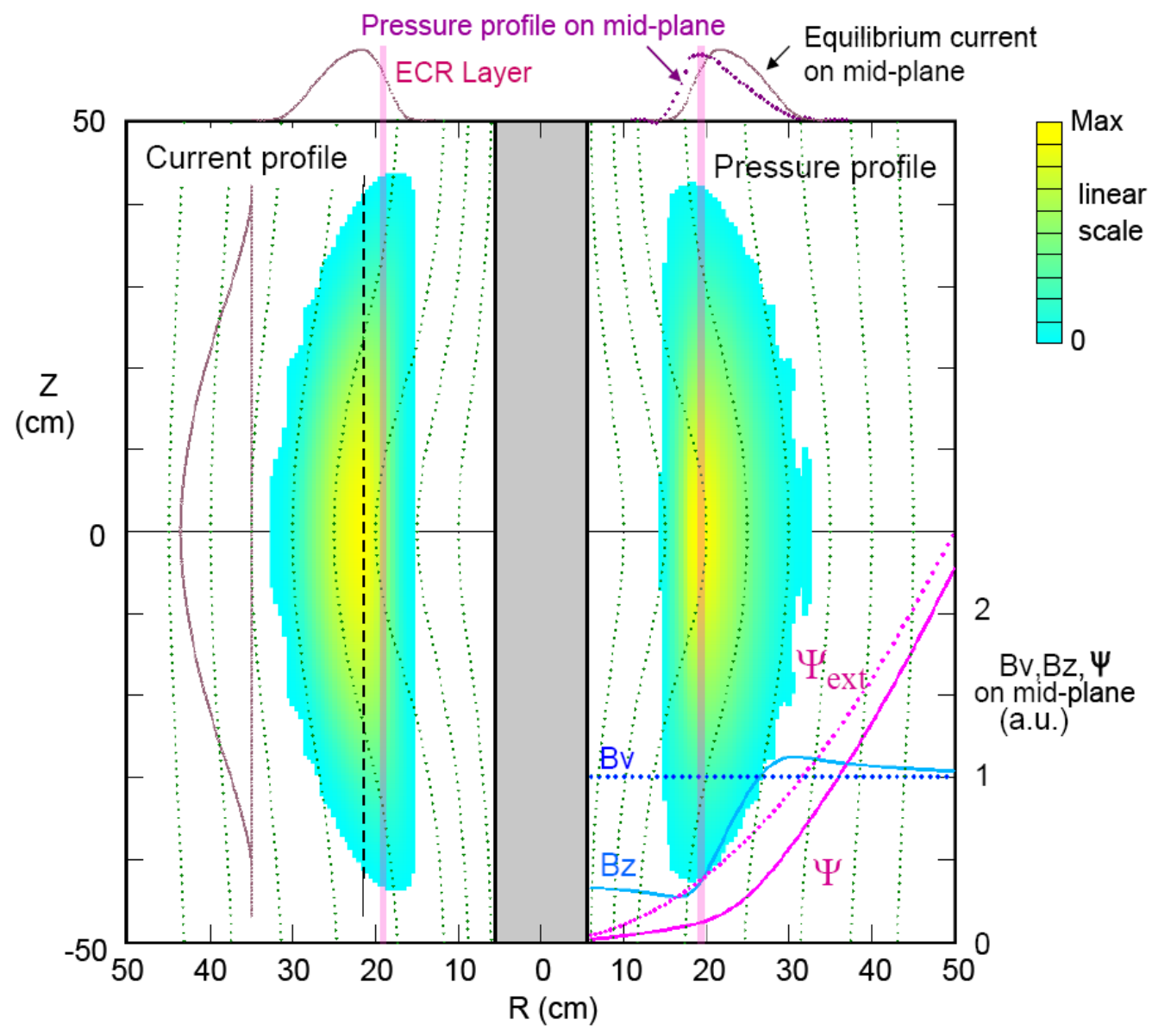

Figure 8. Open field equilibrium modeled for an experiment in the LATE device. $R_{0}=0.24 \mathrm{~m}, a=0.08 \mathrm{~m}, b=0.44 \mathrm{~m}, \alpha=1.8, \kappa=5.5, \delta=0.8, \sigma=-0.3$, $I_{E Q L}=-1100 \mathrm{~A}, B_{V}=30 \mathrm{G}, B_{\phi}=1285 \mathrm{G}$ at $R=25 \mathrm{~cm}, j_{\text {peak }}=27.7$ $\mathrm{kAm}^{-2}, \mathrm{p}_{\text {peak }}=8.5 \mathrm{~Pa}\left(n_{\text {epeak }}=10^{18} \mathrm{~m}^{-3}, \mathrm{~T}_{\text {epeak }}=53 \mathrm{eV}\right)$

for the first step field shown in figure 10. The result is plotted in figure 11. This time a clear closed flux surface which extended to the center post is formed.

In figures 10 and 11 we estimate toroidal currents carried by the energetic electrons in the trapped and passing orbits in the range of $v=v_{0}$ to $v_{c}$. These electrons may be generated by EC heating in a rather narrow zone along the ECR layer. Suppose an energetic confined electron that leaves one of above sample points in this zone. The electron crosses field lines and makes a periodic orbit. We divide the poloidal cross section into fine square cells numbered as $k=1,2,3, \ldots$. We trace out one cycle of the orbit in the poloidal section and find the period $T$ and each span of toroidal angle $\phi_{k}$ upon the traverse through the $\mathrm{k}$-th cell. The toroidal current in the $\mathrm{k}$-th cell contributed by this single electron that has $v$ and $\theta$ at the starting point on the heating zone is given by,

$$
i_{k}(v, \theta)=\frac{e \phi_{k}}{2 \pi T}
$$

This is a kind of "Green function" since by summing-up this elemental contribution 

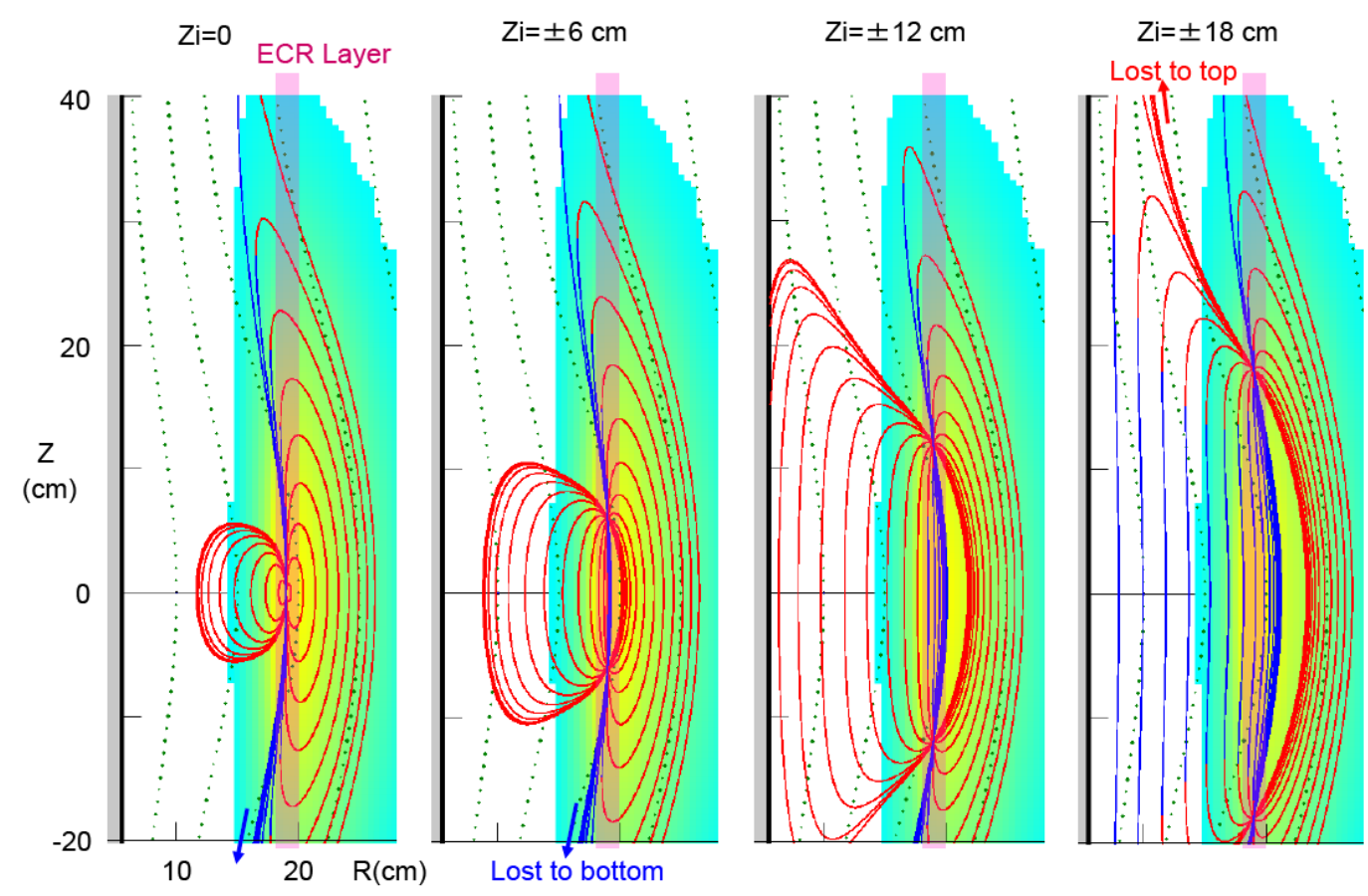

Figure 9. Electron orbits starting from various vertical points on the ECR layer with a velocity $v=2.33 \times 10^{7} \mathrm{~ms}^{-1}$ and pitch angles evenly distributed over 3 to 177 degree by a step of 6 degree. Blue denotes backward drift part in toroidal direction.

over the velocity distribution for the electrons on the confinement mapping, we have the additional current distribution from the confined energetic electrons.

At this point we introduce the lower boundary in velocity range, $v_{0}$, over which the electrons are essentially collision-less in the sense that they can make cross-field passing orbits. Actually, we set $v_{0} \approx 2 v_{t}$, where $v_{t} \equiv \sqrt{2 T_{\text {epeak }} / m_{e}}$ is the electron thermal velocity at the peak position. We also introduce the upper boundary, $v_{c}$, over which the collision time is longer than $6 \mathrm{msec}$ and pitch angle scattering becomes too slow to give significant $v_{\|}$and we neglect contribution from these electrons. For simplicity, we use same $v_{0}$ and $v_{c}$ for each segment of 11 heating sample points.

To meet the lower and upper velocity boundary, we look for the solution that superposes two steady solutions as

$$
f_{s}(v, \theta)=f_{1}(v, \theta)+f_{2}(v, \theta)=g_{1}(v) h_{1}(\theta)+g_{2}(v) h_{2}(\theta)
$$

The lower and upper boundary conditions,

$$
\begin{aligned}
& f_{s}\left(v_{0}, \pi / 2\right)=g_{1}\left(v_{0}\right)+g_{2}\left(v_{0}\right)=\frac{n_{\text {epeak }}}{\left(\sqrt{\pi} v_{t}\right)^{3}} \exp \left[-\left(\frac{v_{0}}{v_{t}}\right)^{2}\right] \\
& f_{s}\left(v_{c}, \pi / 2\right)=g_{1}\left(v_{c}\right)+g_{2}\left(v_{c}\right)=0
\end{aligned}
$$

give $g_{1}(v)=A\left(v_{c} / v\right)^{Q_{1}}$ and $g_{2}(v)=-A\left(v_{c} / v\right)^{Q_{2}}$ with

$$
A=\frac{n_{\text {epeak }}}{\left(\sqrt{\pi} v_{t}\right)^{3}} \exp \left[-\left(\frac{v_{0}}{v_{t}}\right)^{2}\right]\left[\left(\frac{v_{c}}{v_{0}}\right)^{Q_{1}}-\left(\frac{v_{c}}{v_{0}}\right)^{Q_{2}}\right]^{-1} \text {. }
$$




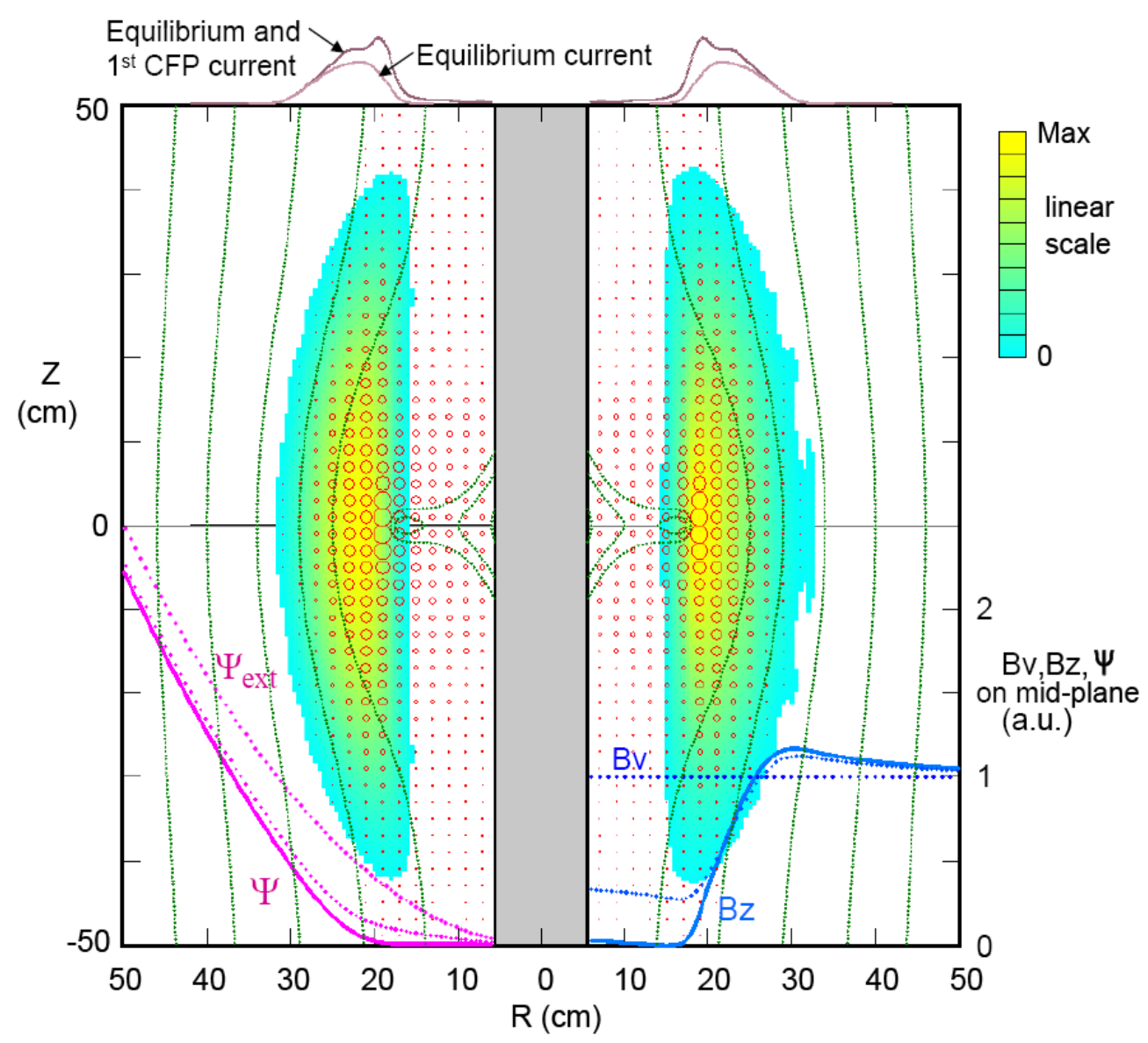

Figure 10. First step field in which contribution from first step CFP current is superposed on pressure driven equilibrium field. $I_{E Q L}=-1100 \mathrm{~A}$ and $I_{C F P}=-367$ A. Area of red circle denote magnitude of CFP current on each square cell $\left(2 \times 2 \mathrm{~cm}^{2}\right)$, $v_{0}=7.7 \times 10^{6} \mathrm{~ms}^{-1}, v_{c}=4.8 \times 10^{7} \mathrm{~ms}^{-1}$, Power to maintain tail $\simeq 2 \mathrm{~kW}$

Then,

$$
f_{s}(v, \pi / 2)=A\left(\frac{v_{c}}{v}\right)^{Q_{1}}\left[1-\left(\frac{v}{v_{c}}\right)^{Q_{1}-Q_{2}}\right]
$$

Here, $Q_{1}(>0)$ is the main parameter that roughly determines the slope of $f_{s}(v, \pi / 2)$ along the EC-wave driven diffusion line. While we set $Q_{2}=Q_{1}-1$ in the calculations other choices such as, for example $Q_{2}=Q_{1}-2$, do not essentially change the results. Upon the change from the single steady solution $f(v, \theta)=g(v) h(\theta)$ to the superposed solution (equation (36)) equation (33) may be generalized as

$$
Q_{e f f}=\sqrt{\frac{\Gamma\left(1+Z_{e f f}\right)}{2 \Delta_{W} f_{s}}\left(\left.\frac{\partial f_{s}}{\partial \theta}\right|_{\theta=\frac{\pi}{2}-}-\left.\frac{\partial f_{s}}{\partial \theta}\right|_{\theta=\frac{\pi}{2}+}\right)}
$$

where, $f_{s}=f_{s}(v, \pi / 2)$ and

$$
Q_{e f f}=-\left.\frac{v}{f_{s}} \frac{\partial f_{s}}{\partial v}\right|_{\theta=\frac{\pi}{2}} .
$$




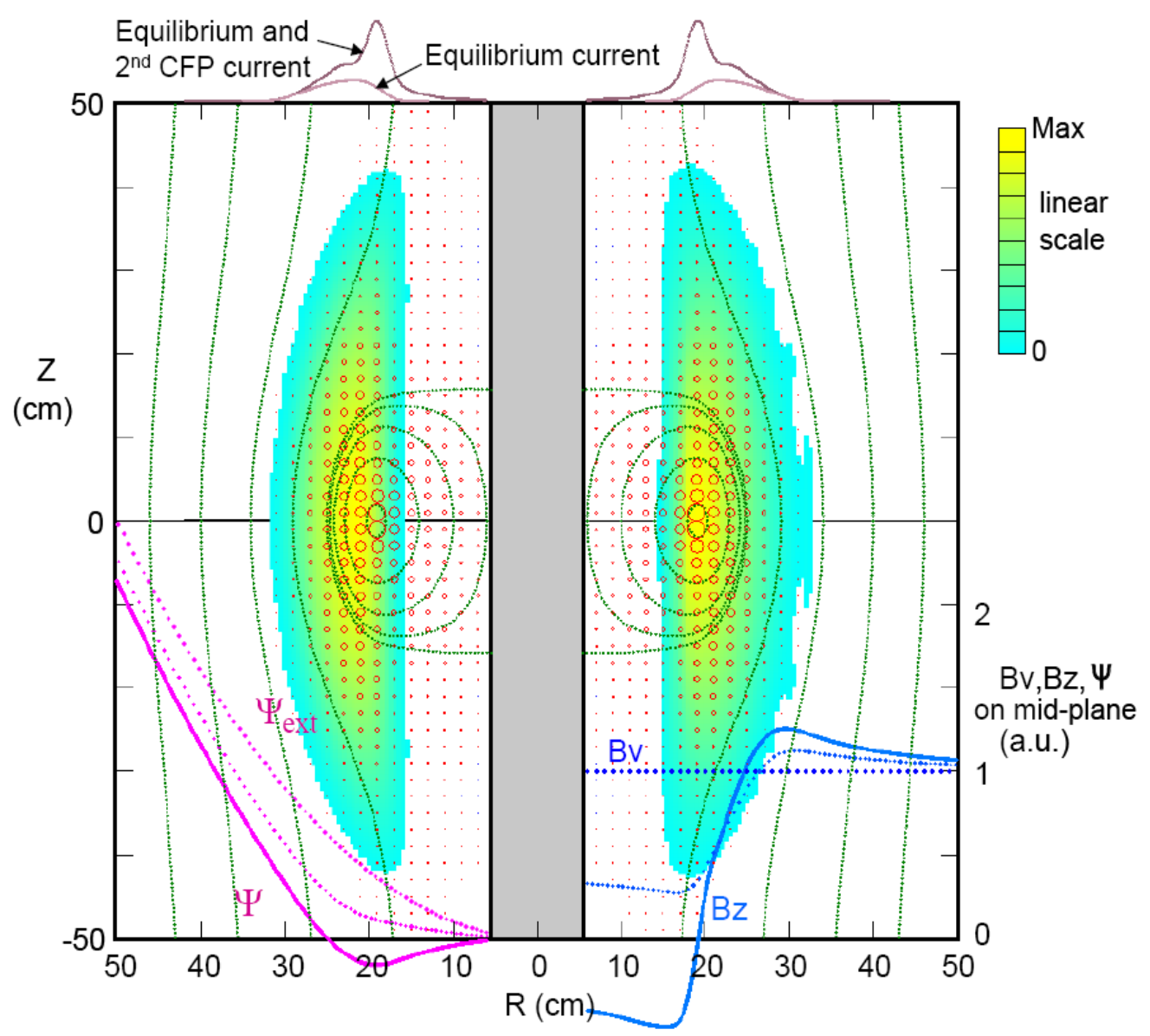

Figure 11. Second step field in which contribution from second step CFP current is superposed on pressure driven equilibrium field. $I_{E Q L}=-1100 \mathrm{~A}$ and $I_{C F P}=-841$ A. Area of red circle denote magnitude of CFP current on each square cell $\left(2 \times 2 \mathrm{~cm}^{2}\right)$, $v_{0}=7.7 \times 10^{6} \mathrm{~ms}^{-1}, v_{c}=4.8 \times 10^{7} \mathrm{~ms}^{-1}$, Power to maintain tail $\simeq 2.3 \mathrm{~kW}$

In the following calculations we control $Q_{1}$ value to simulate the experiments, which determines the superposed solution $f_{s}(v, \theta)$. Then information on $\Delta_{W}$ is obtained from equation (37) as a function of $v$. Thus we do not directly handle $\Delta_{W}$. Equation (32) is still effective for the superposed solutions if $f$ is replaced by $f_{s}$.

The tail electron density $n_{\text {tail }}$ is given by

$$
n_{\text {tail }}=\int_{0}^{\pi} d \theta \int_{v_{0}}^{v_{c}} f_{s}(v, \theta) 2 \pi v^{2} \sin \theta d \theta d v
$$

The tail volume is assumed to be

$$
V_{\text {ol }} \text { tail }=2 \pi R_{E C R} S_{\text {tail }}
$$

Here $R_{E C R}$ is the radius at the ECR layer and we set $S_{\text {tail }}$ to be one third of the pressure cross section by observing the spread of various orbits in figure 9 . We divide the tail 
volume equally into each segment of 11 heating sample points. Total tail number is $N_{\text {tail }}=n_{\text {tail }} V_{\text {ol }}$ tail. The toroidal current carried by energetic electrons in the $\mathrm{k}$-th square cell, $I_{k}$ is given by summing up the contribution from every energetic electron including both CFP and trapped electrons over the confinement mapping

$$
I_{k}=V o l_{\text {tail }} \times \int_{0}^{\pi} d \theta \int_{v_{0}}^{v_{c}} i_{k}(v, \theta) f_{s}(v, \theta) 2 \pi v^{2} \sin \theta d \theta d v
$$

In order to obtain the results in figures 10 and 11 we calculate above procedures for each segment of 11 starting points distributed vertically and sum up the contributions. The results for the segment on the mid-plane $\left(Z_{i}=0\right)$ are shown in figures 12 and 13. Initial confinement mapping have a lost area in forward direction. The lost area is much smaller than the case in figure $5(\mathrm{~b})$ where both the radial coordinate $R_{E C R}$ and the magnitude of $B_{Z}$ at the ECR layer is larger than the present case. This dependence on $R_{E C R}$ and $B_{Z}$ will be discussed later.
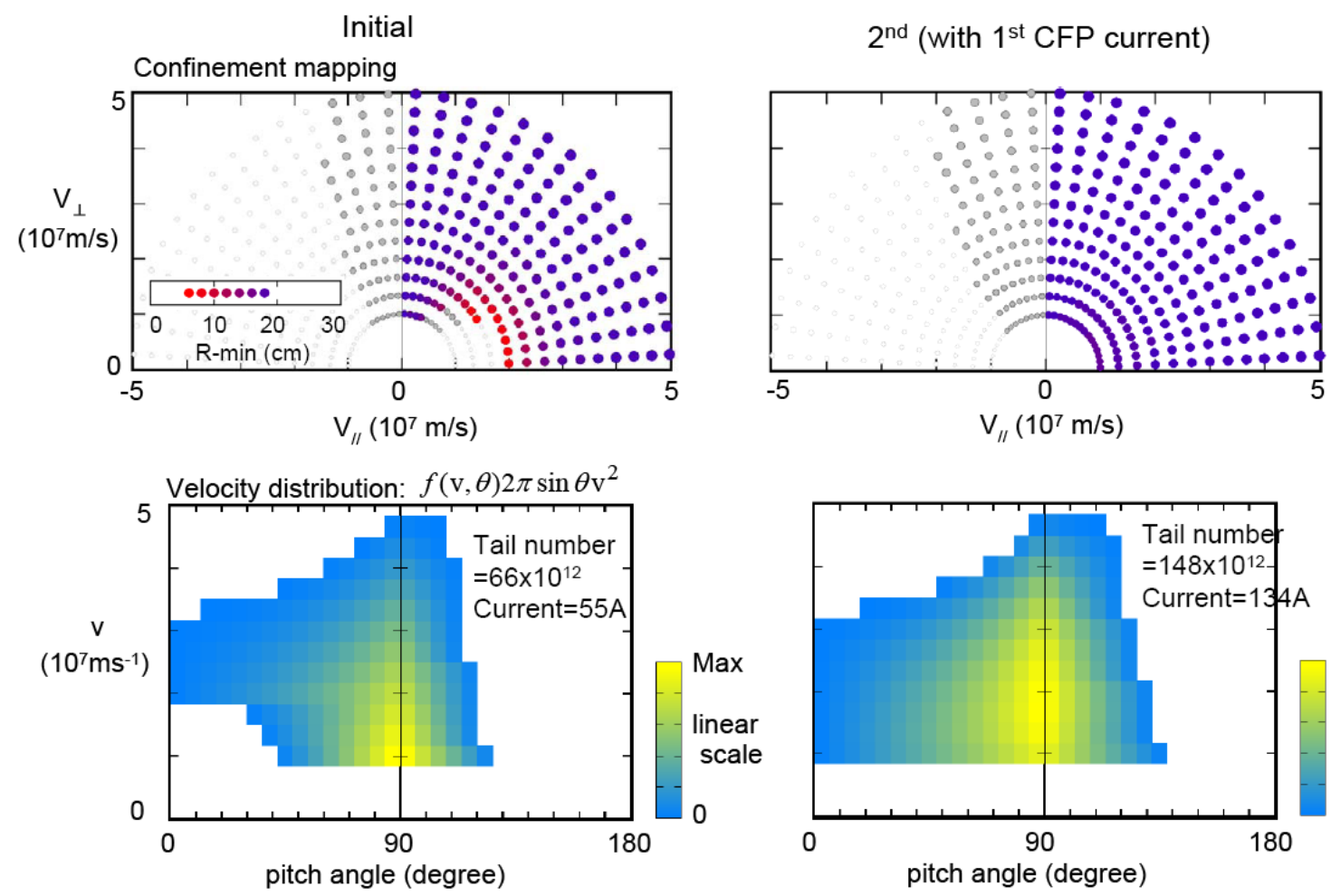

Figure 12. Confinement mappings and weighted velocity distributions for the midplane segment of $Z_{i}=0$. The results for initial (figure 8) and the first step (figure 10) fields are plotted.

With the first-step CFP current, the lost area disappears and all forward area becomes passing domain. At the same time, population of the CFP and trapped electrons as well as the CFP current of this segment increases more than twice in the second step calculations. This result indicates improvement of confinement of CFP 


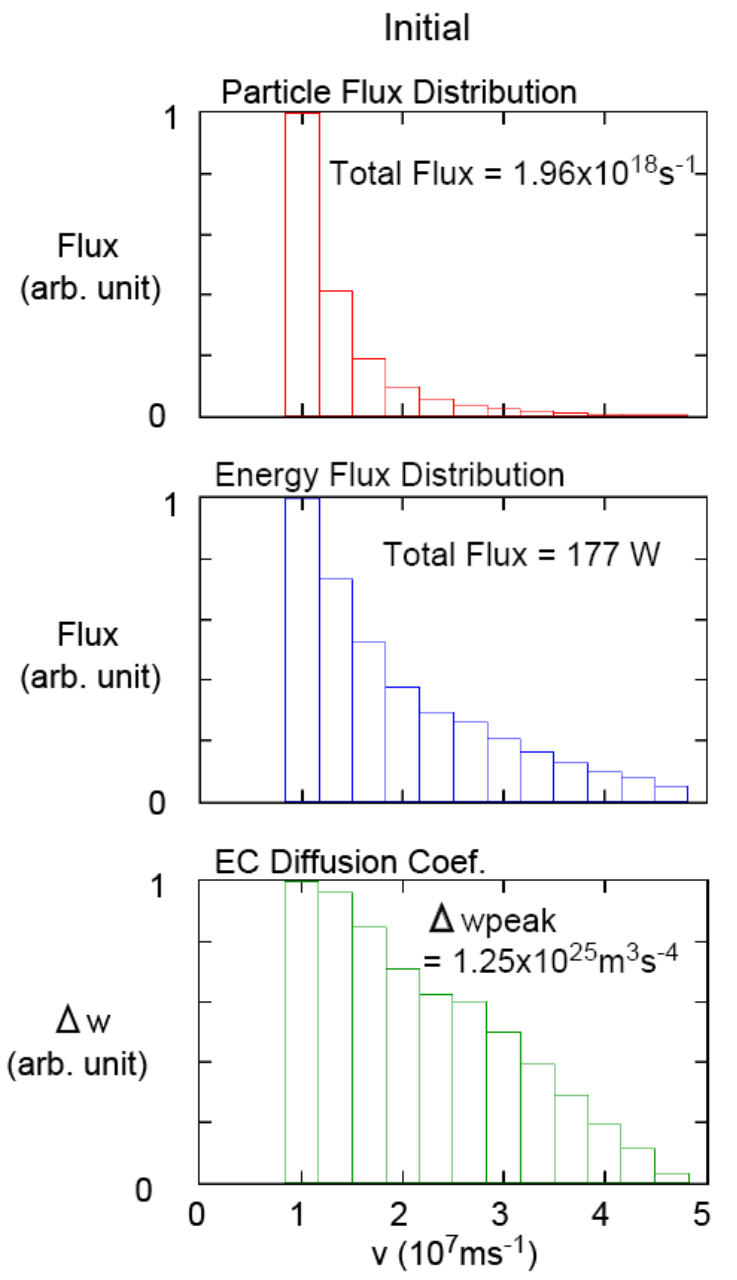

$2^{\text {nd }}$ (with $1^{\text {st }}$ CFP current)
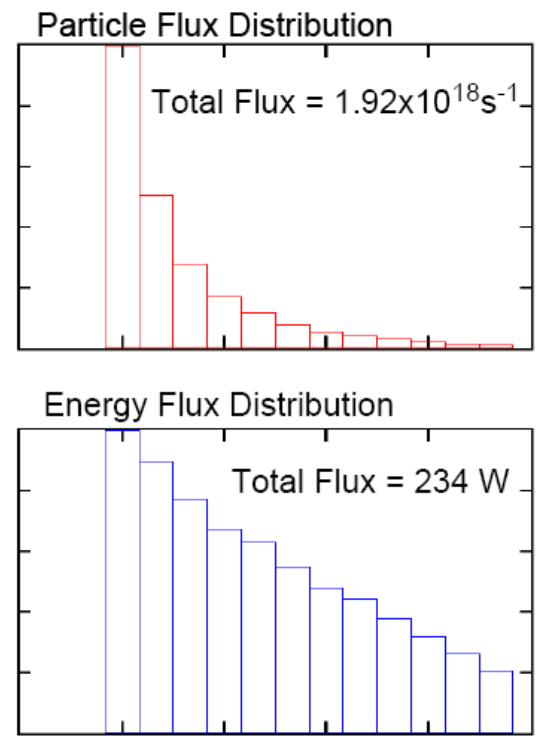

EC Diffusion Coef.

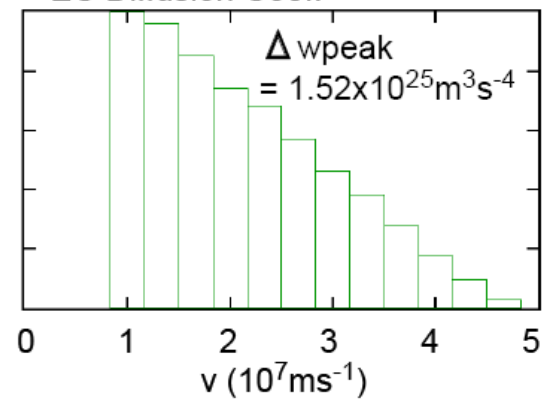

Figure 13. Particle and energy fluxes of EC heated electrons via pitch-angle scattering toward both sides around $\theta=\pi / 2 \mathrm{ECH}$ line and EC-wave diffusion coefficient $\Delta_{W}$ along the ECH line (see figure 7) for the mid-plane segment of $Z_{i}=0$ are shown as functions of velocity. Here $\Delta_{W}$ was calculated using equation (37). The results for initial (figure 8) and the first step (figure 10) fields are plotted.

electrons due to the fact that particle loss via pitch angle diffusion to the loss area in the forward direction disappears in the second step. Increase of the current is mainly due to increase of total CFP electrons on whole forward confinement area in the velocity space by the improvement of confinement. Contribution from the current carried by the portion of CFP electrons that fill up initially lost area is not significant. Here we adjusted $Q_{1}$ value so that the particle flux in each segment both in initial and second steps became almost the same $\left(\approx 2 \times 10^{18} \mathrm{~s}^{-1}\right.$ in the present case). The $Q_{1}$ was adjusted to be 2 and 1.4 for the mid-plane segment in initial and second step calculations, respectively and the $Q_{1}$ value was adjusted larger as the segment was away from the mid-plane. On the other hand the $\Delta_{W}$ spectra are found to be nearly the same for every segment both in initial and the second steps. The $\Delta_{W}$ spectra decrease with $v$ in figure 13, which may correspond to the experimental situation where EC-waves having a wide $N_{\|}$spectrum 
from zero to unity generate CFP electrons, as inferred from the discussion for $\Delta_{W}$ in section 4 and the discussion for EC diffusion in section 7 .

Above results presented in figures 8-13 may explain the LATE experiment where an initial closed flux surface is generated via current jump from $\approx 1 \mathrm{kA}$ to $\approx 2 \mathrm{kA}$ by 24 $\mathrm{kW}$ microwave power injected under $B_{V}=30 \mathrm{G}[6]$. The present model predicts $2.4 \mathrm{~kW}$ to maintain the current carrying energetic electrons. This is $10 \%$ of the injection power in the experiment. Two facts may explain this discrepancy. First of all much of the power is still required to support the relative large current outside the closed flux surface region as well as to support the pressure driven plasma itself. Second, as shown in figure 9, EC heating at $Z>18 \mathrm{~cm}$ and $Z<-18 \mathrm{~cm}$ hardly contributes the current. Therefore we do not include contribution from $Z>20 \mathrm{~cm}$ and $Z<-20 \mathrm{~cm}$. The absorption power in these zones is not took account into in the model. When we take into account these points, the $10 \%$ result looks fairly reasonable.

Let us discuss this comparison between the model and the experiment from another point. First note that the power and the additional current by CFP electrons increases when particle flux is increased by decreasing $Q_{1}$ value for steady solutions (equation $(36)$ ). While the particle flux value is a free parameter in the model, the used value $\left(\approx 2 \times 10^{18} s^{-1}\right.$ in the present case) has a reality if the $10 \%$ result is reasonable as described above. Since it is difficult to estimate the EC wave diffusion coefficient in experiments, present approach in which a comparison in terms of microwave power and driven current is made between the experiment and the model using appropriate steady solutions is useful.

Here, it is noted that as far as $B_{\phi}>>B_{V}$, magnitude of $B_{\phi}$ does not affect all results presented in figures $8-13$, where $B_{\phi}=1285$ Gauss is nominally employed. This is because characteristics of drift motion described by equations (16)-(24) and equilibrium equation (12) essentially do not depend on the magnitude of $B_{\phi}$ as far as $B_{\phi}>>B_{V}$. Especially, we employ $B_{\phi}=480$ Gauss in figure 6 in order to see explicitly electron Larmor motions.

\section{Case in a large tokamak}

We examine the model described in sections 2-4 for experiments in large tokamaks of DIII-D and JT-60U [11, 12]. Modeled open field equilibrium is shown in figure 14. We adopt 9 sample heating points along the ECR layer $\left(Z_{i}=\right.$ $-0.2,-0.15,-0.1,-0.05,0,0.05,0.1,0.15$ and $0.2 \mathrm{~m}$ at $\left.R_{i}=2.61 \mathrm{~m}\right)$ as the starting points of orbits as partly shown in figure 15 and add each contribution to obtain the CFP current profile. Detailed procedure to obtain the CFP current profile is the same as the case in section 5. This time, however, we set $S_{\text {tail }}$ to be one fourth of the pressure cross section by observing the spread of various orbits in figure 15 and we also adjust $Q_{1}$ value so that the particle flux in each segment in initial and second steps becomes almost the same for all segments $\left(\approx 3.8 \times 10^{20} s^{-1}\right.$ this time). The result is shown in figure 16 , where $B_{Z}$ is lowered beyond zero and a quite small negative $B_{Z}$ area appears. Next, we 
adopt the same sample heating points as the starting points of orbits and calculate the second step CFP current for the first step field shown in figure 16. The result is plotted in figure 17, where a small but clear closed flux surface is formed.

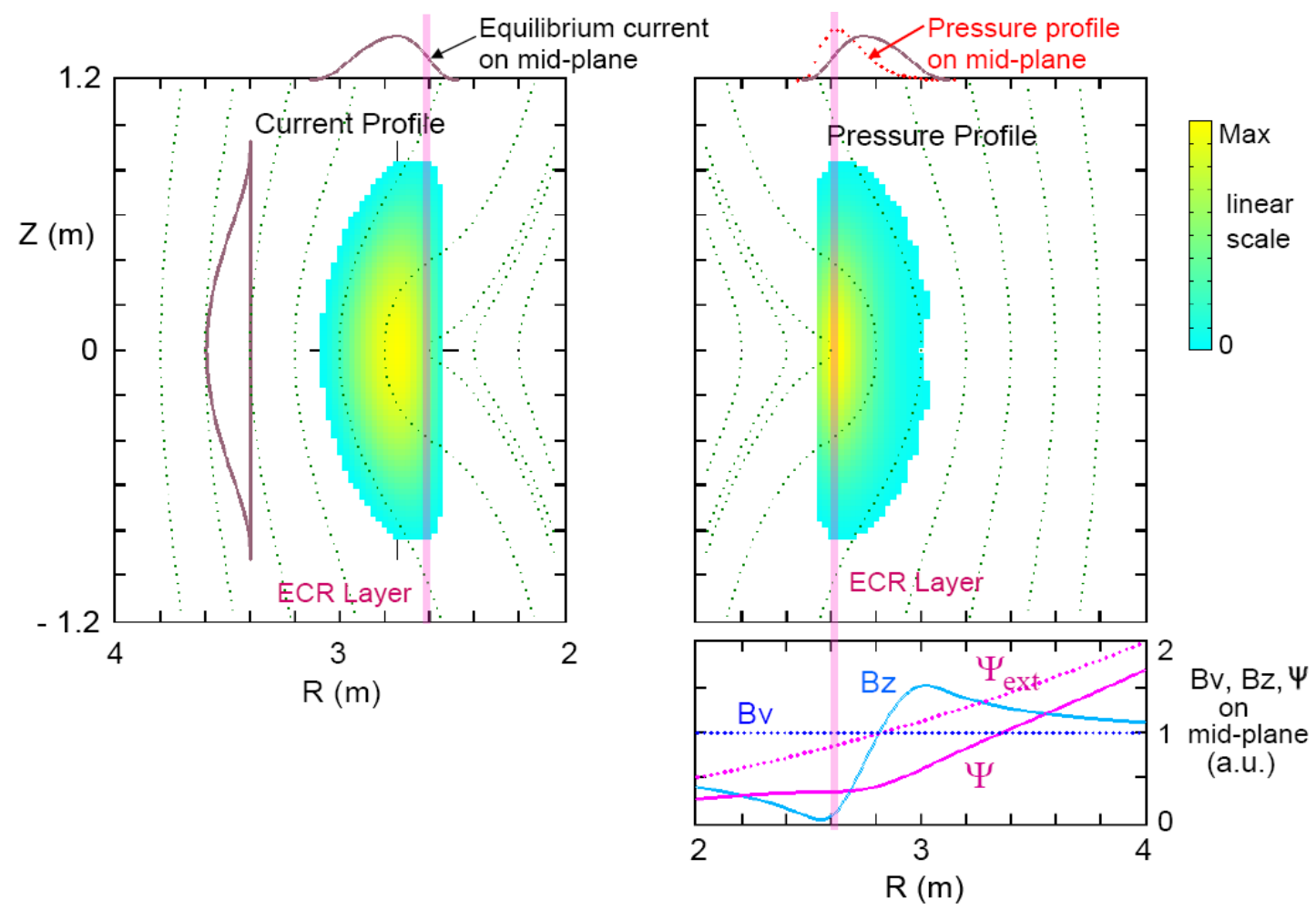

Figure 14. Open field equilibrium modeled for experiments in large tokamaks. $R_{0}=2.8 \mathrm{~m}, a=0.3 \mathrm{~m}, b=0.9 \mathrm{~m}, \alpha=1.8, \kappa=3, \delta=0.5, \sigma=-0.2, I_{E Q L}=-5000 \mathrm{~A}, B_{V}=30$ $\mathrm{G}, B_{\phi}=34200 \mathrm{G}$ at $R=3 \mathrm{~m}, j_{\text {peak }}=16.3 \mathrm{kAm}^{-2}$, $\mathrm{p}_{\text {peak }}=82.6 \mathrm{~Pa}\left(n_{\text {epeak }}=8 \times 10^{18}\right.$ $\left.\mathrm{m}^{-3}, \mathrm{~T}_{\text {epeak }}=65 \mathrm{eV}\right)$

Figure 18(a) shows the confinement mapping for the starting point of $R_{i}=2.61 \mathrm{~m}$ and $Z_{i}=0 \mathrm{~m}$ in the initial equilibrium field shown in figure 14. A quite large difference is seen compared with the cases in the small device shown in figures 5 (b) and 12, that is, although inside $B_{Z}$ is suppressed by the equilibrium current almost to the limit of open to closed field in figure 14, there remains a large hole of lost area in the forward direction in the confinement mapping in velocity space. The CFP orbits quickly disappear as the starting point shifts vertically apart from the mid-plane as seen in figure 15 . Therefore there appears only a small CFP current in a limited area around the mid-plane in figure 16. We need quite a large power $\approx 140 \mathrm{~kW}$ to keep this small CFP current in the present case since the loss via the pitch angle diffusion is very fast due to the large hole of lost area even in the mid-plane segment, much larger loss in off mid-plane segments. This power is $\approx 10 \%$ of the power injected in the experiments $[11,12]$ and seems to be rather reasonable when we take into account the same reasons as those mentioned in the previous section.

While this first step CFP current is small, it is rather localized and produce a quite 


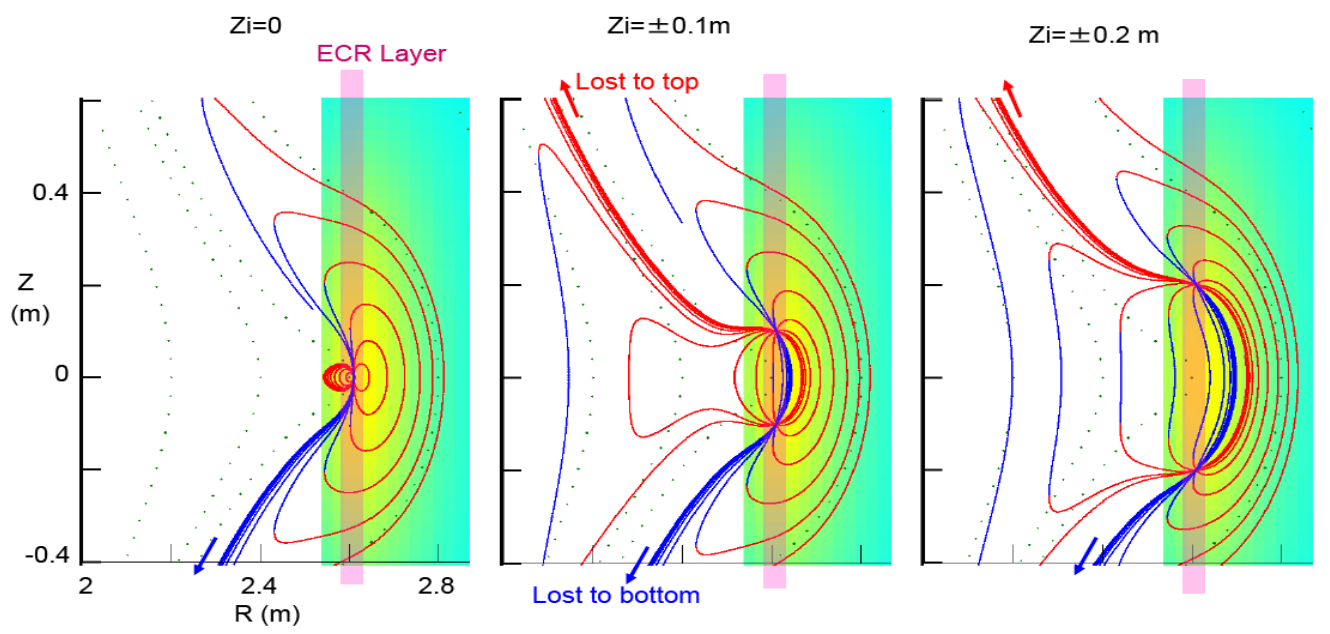

Figure 15. Electron orbits starting from various vertical points on ECR layer with a velocity $v=6.67 \times 10^{7} \mathrm{~ms}^{-1}$ and pitch angles evenly distributed over 3 to 177 degree by a step of 6 degree. Blue denotes backward drift in toroidal direction.

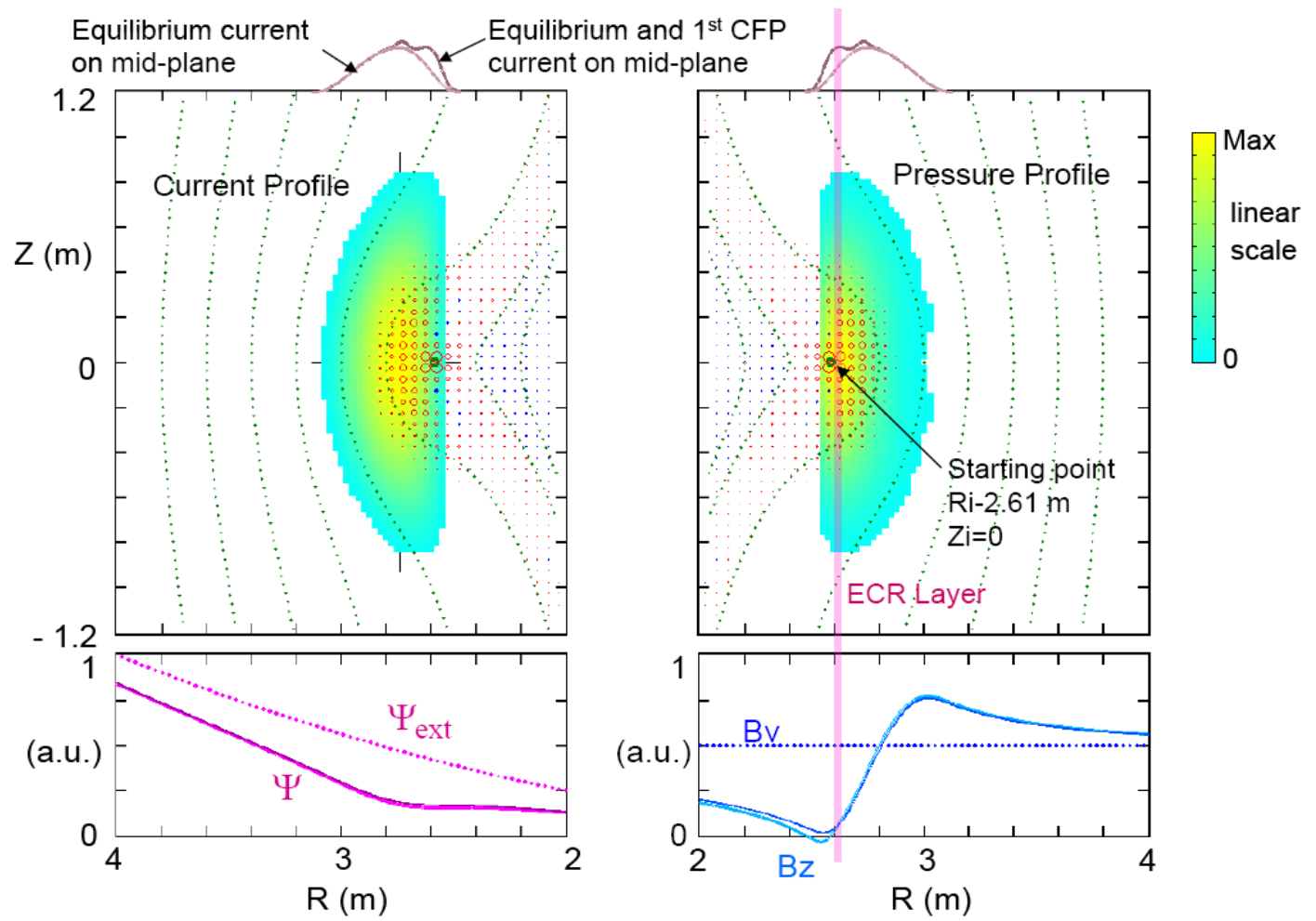

Figure 16. First step field in which contribution from first step CFP current is superposed on pressure driven equilibrium field. $I_{E Q L}=-5000 \mathrm{~A}$ and $I_{C F P}=-229$ A. Area of circles denotes magnitude of CFP current on each square cell $\left(5 \times 5 \mathrm{~cm}^{2}\right)$. Red (Blue) circle denotes forward (backward) current. $v_{0}=1.15 \times 10^{7} \mathrm{~ms}^{-1}, v_{c}=$ $9.67 \times 10^{7} \mathrm{~ms}^{-1}$. Power to maintain tail $\simeq 140 \mathrm{~kW}$ 


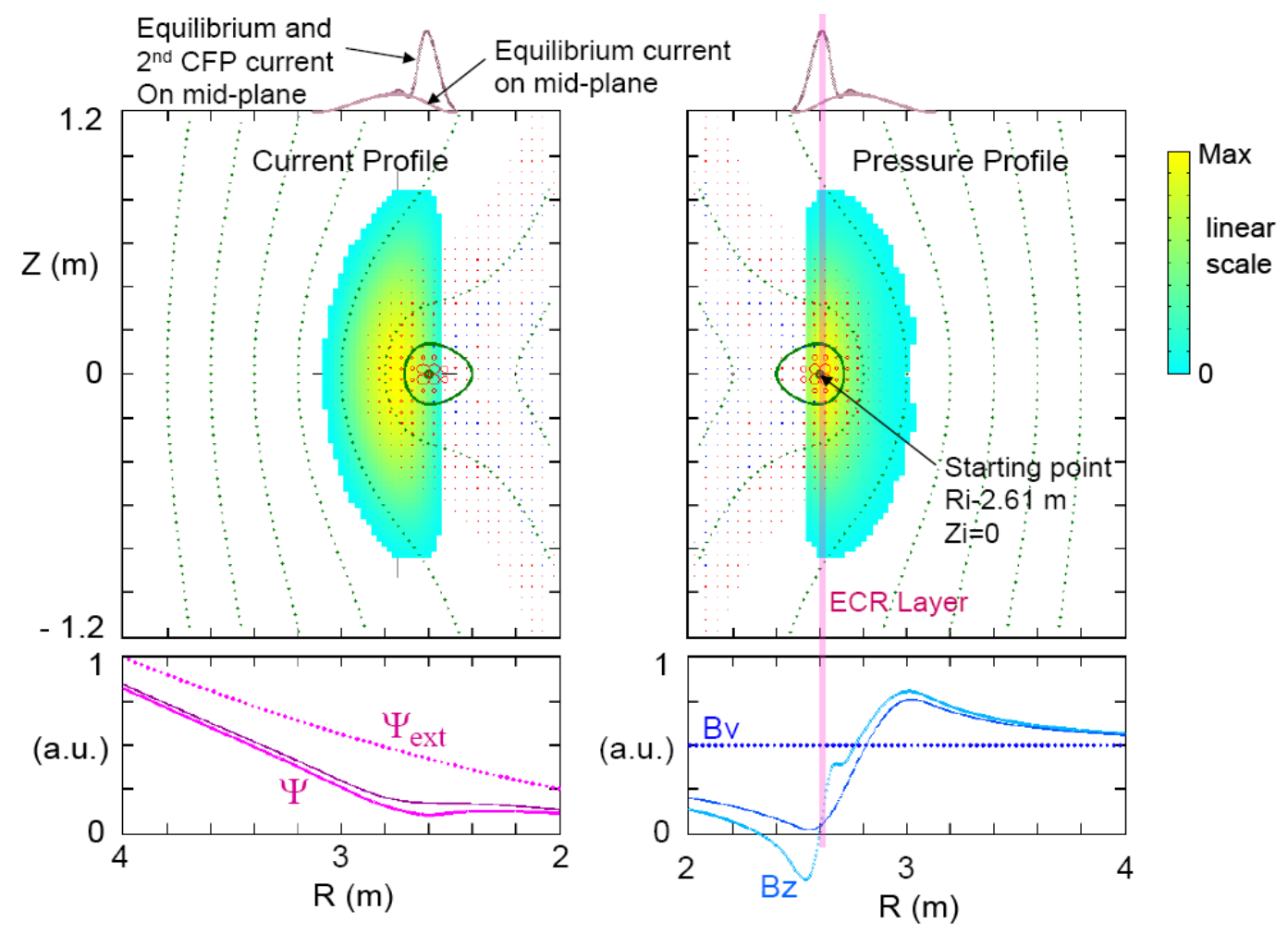

Figure 17. Second step field in which contribution from second step CFP current is superposed on pressure driven equilibrium field. $I_{E Q L}=-5000 \mathrm{~A}$ and $I_{C F P}=-822$ A. Area of circles denotes magnitude of CFP current on each square cell $\left(5 \times 5 \mathrm{~cm}^{2}\right)$. Red (Blue) circle denotes forward (backward) current. $v_{0}=1.15 \times 10^{7} \mathrm{~ms}^{-1}, v_{c}=$ $9.67 \times 10^{7} \mathrm{~ms}^{-1}$. Power to maintain tail $\simeq 150 \mathrm{~kW}$

small negative $B_{Z}$ area. With this small negative area whole forward area becomes CFP domain for the starting point in the mid-plane segment as shown in figure 18(b).

These characteristics in the large device are quite different from those in small device. To explain this difference we trace out how $v_{\|}$changes in a CFP orbit to conserve three constants of motion (equations (16)-(18)) by supposing that an electron moves from $R=R_{i}+\Delta$ and $Z=0$ to $R=R_{i}$ and $Z=0$ by a short step $\Delta$ via a CFP orbit above or below the mid-plane. Both $B_{\phi}$ and $v_{\perp}^{2}$ increases by $\Delta / R$ to conserve magnetic moment $\mu$. Then $v_{\|}$decreases by $\Delta / 2 R$ to conserve energy. Finally, the $v_{\|}$ condition for conservation of angular momentum is written as

$$
v_{\|} \cong \frac{e R\left[2 B_{Z}+\Delta\left(d B_{Z} / d R\right)\right]}{3 m} \approx \frac{2 e R B_{Z}}{3 m}
$$

This result indicates that $v_{\|}$is approximately proportional to $R B_{Z}$ and that when $R$ is large $v_{\|}$should be increased to make a CFP orbit of the same step $\Delta$. When $\mathrm{R}$ becomes twice, the electron that has the same $v_{\|}$can make a CFP orbit of the same step $\Delta$ if $B_{Z}$ is decreased to half. In order to realize CFP orbits at appropriate energy 

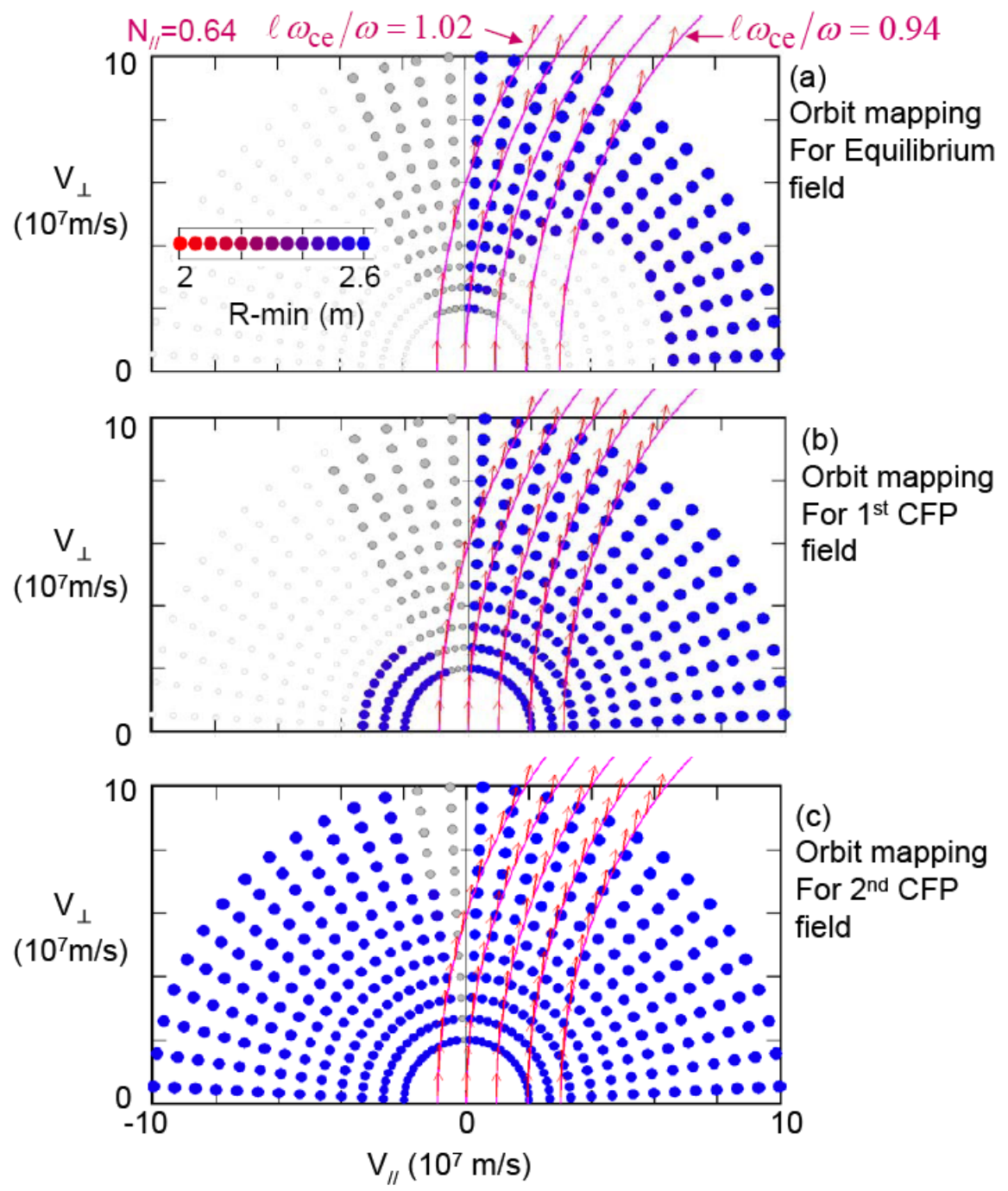

Figure 18. Confinement mapping for starting point $R_{i}=2.61 \mathrm{~m}$ and $Z_{i}=0 \mathrm{~m}$ for (a) initial equilibrium field (figure 14), (b) first step CFP field (figure 16) and (c) second step CFP field (figure 17). EC resonance ellipses for with quasi-linear diffusion arrows for $N_{\|}=0.64$ case at various locations near ECR layer are also plotted. The labeling of the resonance ellipses is explained in Section 7.

range in large devices $B_{Z}$ should be quite lowered. This $R B_{Z}$ scaling was shown in a different way in a previous paper [29].

This scaling predicts that in large devices the equilibrium current should be increased up to the limit of open to closed field equilibrium in order to have effective CFP orbits. We need increase bulk electron pressure to increase the equilibrium current. Fortunately this is relatively straightforward if we can inject sufficient EC power since EC frequency is high and the plasma cutoff density is high in large devices. For example, the peak density is only $6 \%$ of the cut off density in figure 14 .

Suppose that $B_{V}$ is increased twice, for example, in figure 14. Local equilibrium equation (12) as well as the generalized Shafranov formula (15) predicts that when the 
pressure is increased four times, the current density increases twice and the same field lines are obtained.

\section{Appropriate ECH/ECCD for Large Tokamaks}

The results for the large tokamak case shown in figures 14-18 reveal multi roles of EC waves for the generation of a takamak plasma from open field equilibriums.

First is the role of driving sufficient equilibrium current in open fields. EC waves must produce sufficiently high pressure plasma to generate a large equilibrium toroidal current to reduce $B_{Z}$ to almost zero. Second is the pumping-up role. EC waves must pump up electrons perpendicularly in velocity space from bulk to energetic region. Third is the ECCD role. Once an initial small closed flux surface is formed, EC waves must increase the current inside the flux surface by usual ECCD. Here we need careful steering of the beam of EC waves for ECCD onto the small closed flux area as well as appropriate steering of other beams for heating the open field area to keep the equilibrium current. As the area of closed flux surface expands, open field equilibrium current may be replaced one after another by the EC driven current. The wave injections must be adjustable to this expansion of the closed flux surface.

To examine above roles of EC waves, let us start by reviewing basic characteristics of the ECR. The resonance electrons gain the energy $d \epsilon$ and the parallel momentum $d p_{\|}$ at the ratio,

$$
\frac{d p_{\|}}{d \epsilon}=\frac{k_{\|}}{\omega}=\frac{N_{\|}}{c},
$$

at the ECR condition,

$$
1-N_{\|} \frac{v_{\|}}{c}=\frac{\ell \omega_{c e}}{\gamma \omega} \quad(\ell=1,2, . .) .
$$

Here, $\omega_{c e}=e B / m_{e}$ is the EC frequency, $N_{\|}$is the refractive index parallel to the magnetic field and $c$ is the speed of light.

Equation (41) gives the direction of step of the resonance electrons in velocity space upon ECR, which is written using $x=v_{\|} / c$ and $y=v_{\perp} / c$ as follow

$$
\frac{d y}{d x}=\frac{1-N_{\|} x-y^{2}}{\left(N_{\|}-x\right) y} \cong \frac{1-N_{\|} x}{N_{\|} y} \quad(x, y<<1) .
$$

The resonance relation (42) makes an ellipsis in electron velocity space, being referred to as ECR ellipsis. Note that when $\ell \omega_{c e} / \omega=1$ the ellipsis is approximated by $v_{\|} \cong v_{\perp}^{2} / 2 c N_{\|}$ for the region $v_{\|}<<c$.

Figure 19 shows two examples of ECR ellipses on the confinement mapping for the initial equilibrium field in figure 14 . In the case of $N_{\|}=0.87$ the ellipses near the ECR layer approximately realize the perpendicular EC diffusion in figure 7 . The arrows on 

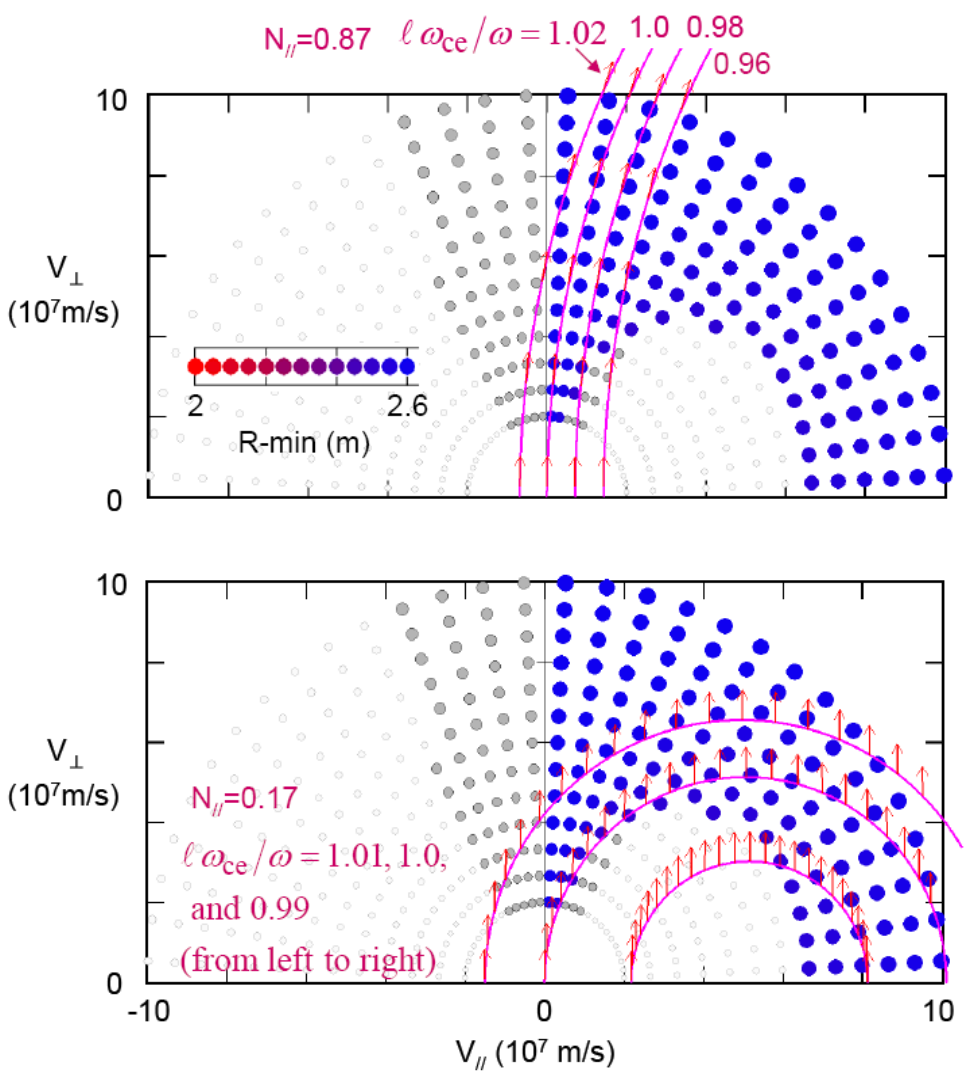

Figure 19. Confinement mapping for starting point $R_{i}=2.61 \mathrm{~m}$ and $Z_{i}=0 \mathrm{~m}$ for initial equilibrium field (figure 13), EC resonance ellipses for with quasi-linear diffusion arrows at various locations near ECR layer for $N_{\|}=0.17$ and 0.87 cases are also plotted.

the ellipses show directions of diffusion given by equation (43). In this high $N_{\|}$case the arrows are approximately tangential to ellipses, which provides an efficient diffusion along the ellipses. Furthermore over a wide range from $\omega_{c e} / \omega=0.98$ to 1.02 the ellipses fulfilled the EC diffusion condition in figure 7, where bulk electrons can be pump up along the lines of resonance ellipses. Note that when $N_{\|}=1$, complete cyclotron auto resonance occurs, where the arrows are exactly tangential to resonance ellipses. On the other hand, the situation is quite different when $N_{\|}$is low (see the $N_{\|}=0.17$ case in figure 19). Thus the pumping-up role is realized only when we use sufficiently high $N_{\|}$ waves.

The equilibrium current drive role and the ECCD role require sufficient absorption of the waves by bulk and tail electrons, respectively. To see how much high $N_{\|}$waves are EC-absorbed, we examine trajectories and optical depths of the waves in the case of oblique O-mode injection at a fundamental resonance frequency and in the case of oblique X-mode injection at a second harmonic resonance frequency, in figures (20) and (22), respectively.

In figure 20 an $\mathrm{O}$-wave is injected on the mid-plane from the outboard with an angle $\theta_{i n j}=25$ degree to the perpendicular direction at the injection point $R_{i n j}=4$ 

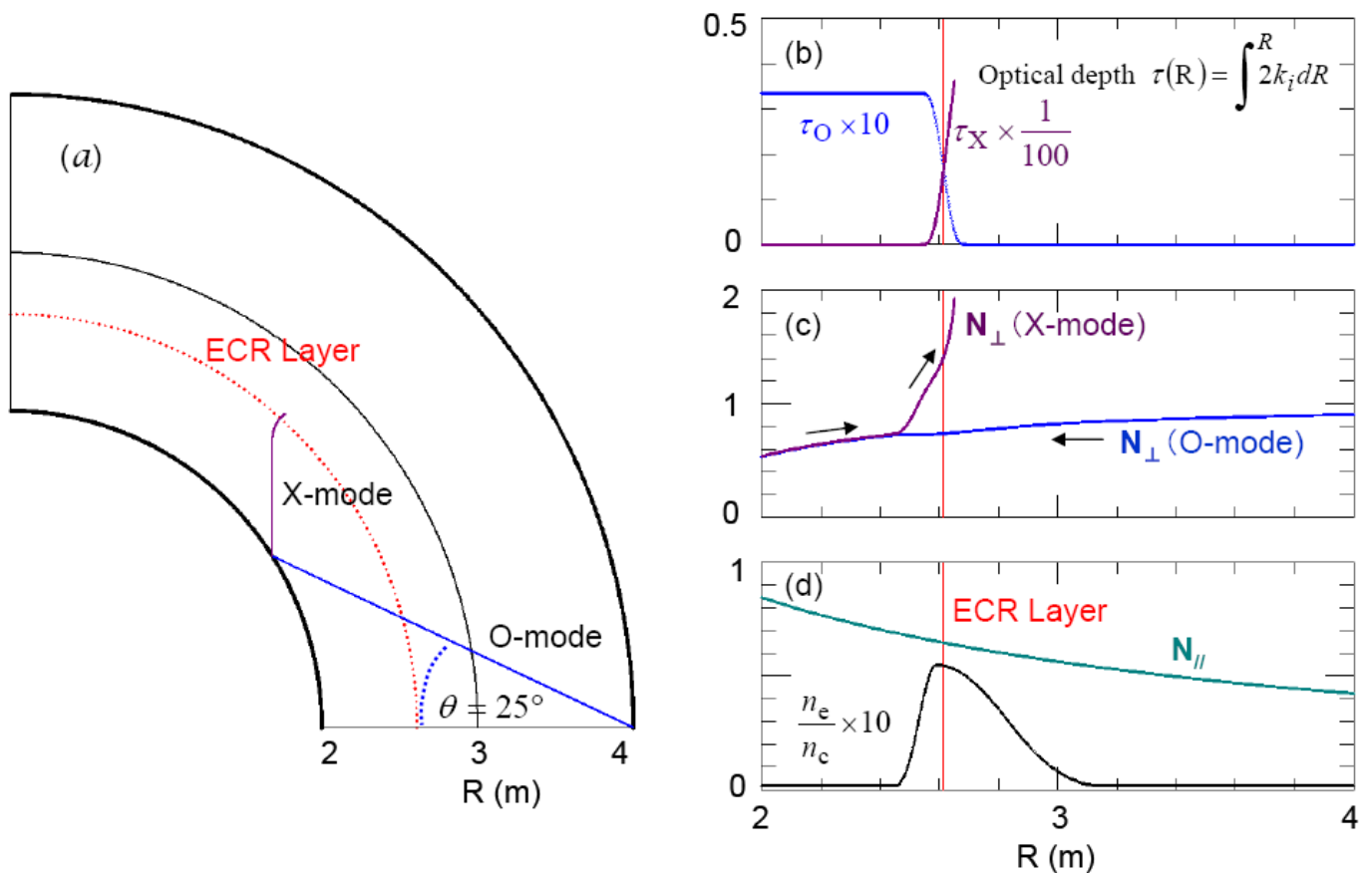

Figure 20. (a) Wave trajectory, (b) optical depth, (c) refractive index and (d) electron density profile for the case of fundamental O-mode injection on the mid plane. Electron temperature $(65 \mathrm{eV})$ is uniform. frequency $=110 \mathrm{GHz}$

m. While the initial refractive index is $N_{\| i n j}=\sin \theta_{i n j} \cong 0.42$, it increases inversely proportional to $R$ as the wave propagates inboard so as to conserve the toroidal mode number $m_{\phi}=R k_{\phi}$ with $N_{\|}=c k_{\phi} / \omega$

$$
N_{\|}=\frac{R_{i n j} N_{\| i n j}}{R}
$$

When the wave propagates towards inboard and attains a relatively high value of $N_{\|}=0.64$ on the ECR layer at $R=R_{E C R}=2.61 \mathrm{~m}$. Almost all injected power penetrates through the ECR layer since the optical depth of oblique O-wave is quite thin $\left(\tau_{O} \cong 0.03\right)$ and reaches the inboard wall and is mirror-reflected on the surface. As shown in figure 21, upon the mirror-reflection with $N_{\|}=0.85$ on the inboard wall over $90 \%$ of the O-mode power is mode-converted into an $\mathrm{X}$-mode power and the $\mathrm{X}$-wave propagates back to the ECR layer and is quite strongly absorbed before reaching the ECR layer since this oblique X mode is essentially right-handed to the toroidal field. We used the method in [30] to calculate the trajectories and absorption of these waves at the fundamental resonance. To obtain the mode conversion rate in figure 21 , we use the formula for the polarizations of oblique $\mathrm{O}$ and $\mathrm{X}$ waves at the plasma boundary [31].

In figure 22 an $\mathrm{X}$-wave at the second harmonic frequency is injected as the same way as the O-wave case in figure 20 . About $20 \%$ of injected power is absorbed even in this low density and low temperature plasma since this oblique $\mathrm{X}$-wave is also essentially righthanded to the toroidal field. We used the method in ref [32] to calculate the trajectories 


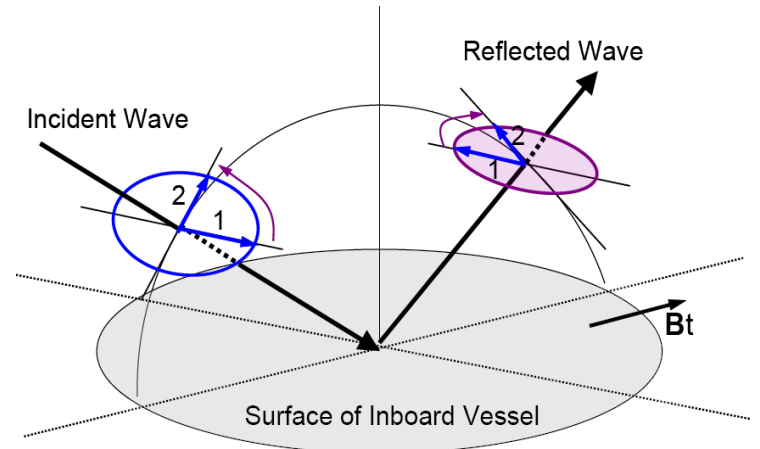

E vectors of Incident and reflected waves rotate opposite to cancel out tangential $\mathrm{E}$ component on the vessel surface.

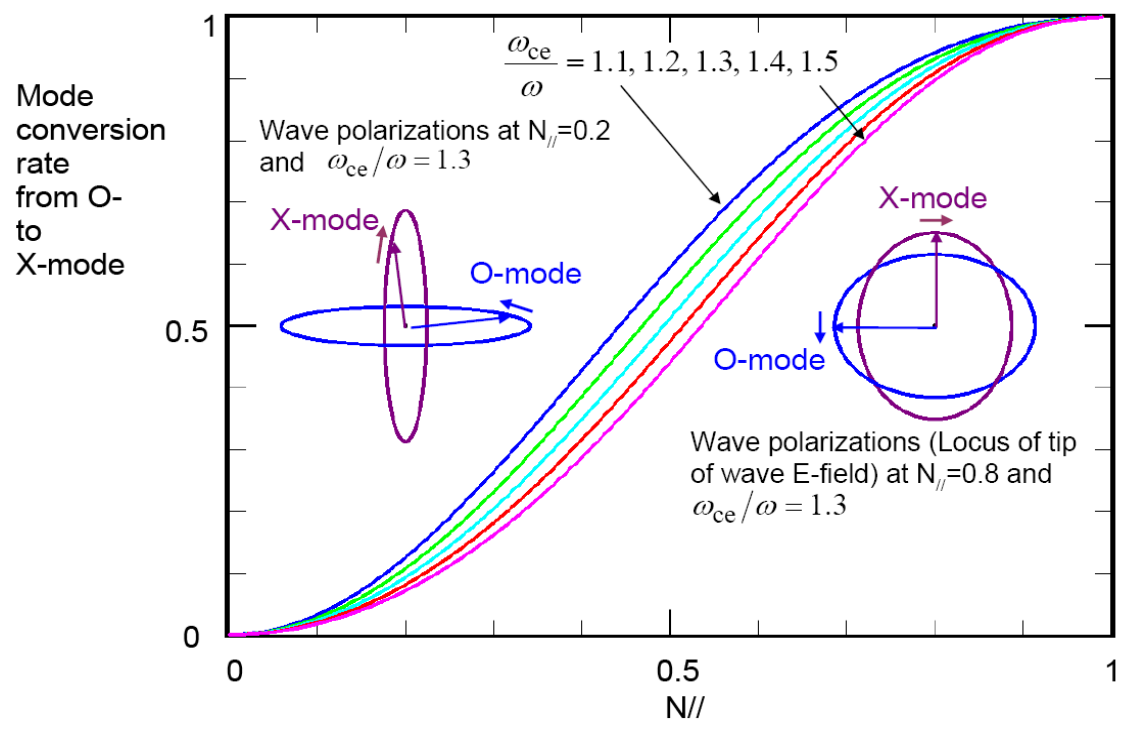

Figure 21. Mode conversion rate from O- to X-wave upon the reflection on a flat mirror. The toroidal field is along the line of intersection of the plane of incidence and the mirror plane.

and absorption of $\mathrm{X}$-waves at the second harmonic resonance, where the absorption is estimated by calculating the quasi-linear diffusion of electrons over the ECR ellipsis in velocity space using the polarization of the cold plasma wave approximation.

Figure 18 shows how these $N_{\|}=0.64$ waves drive electrons on the confinement mapping in velocity space in the successive stages of the initial equilibrium field, the first step CFP field and the second CFP field. In the initial equilibrium field and the first CFP field (figure 18 (a) and (b)), the waves pump up electrons to form a unidirectional tail via pitch-angle scattering to the preferential confinement wing of the CFP orbits. In the second CFP field (figure 18(c)) the preferential confinement disappears and, therefore, the waves should work as an ECCD driver. When the waves approach to the ECR layer from outboard, the ECR ellipsis first appears on the forward wing at relatively high $v_{\|}$region in velocity space as shown in figure 18 (c), which is advantageous for efficient ECCD. When, on the other hand, the waves approach to the 

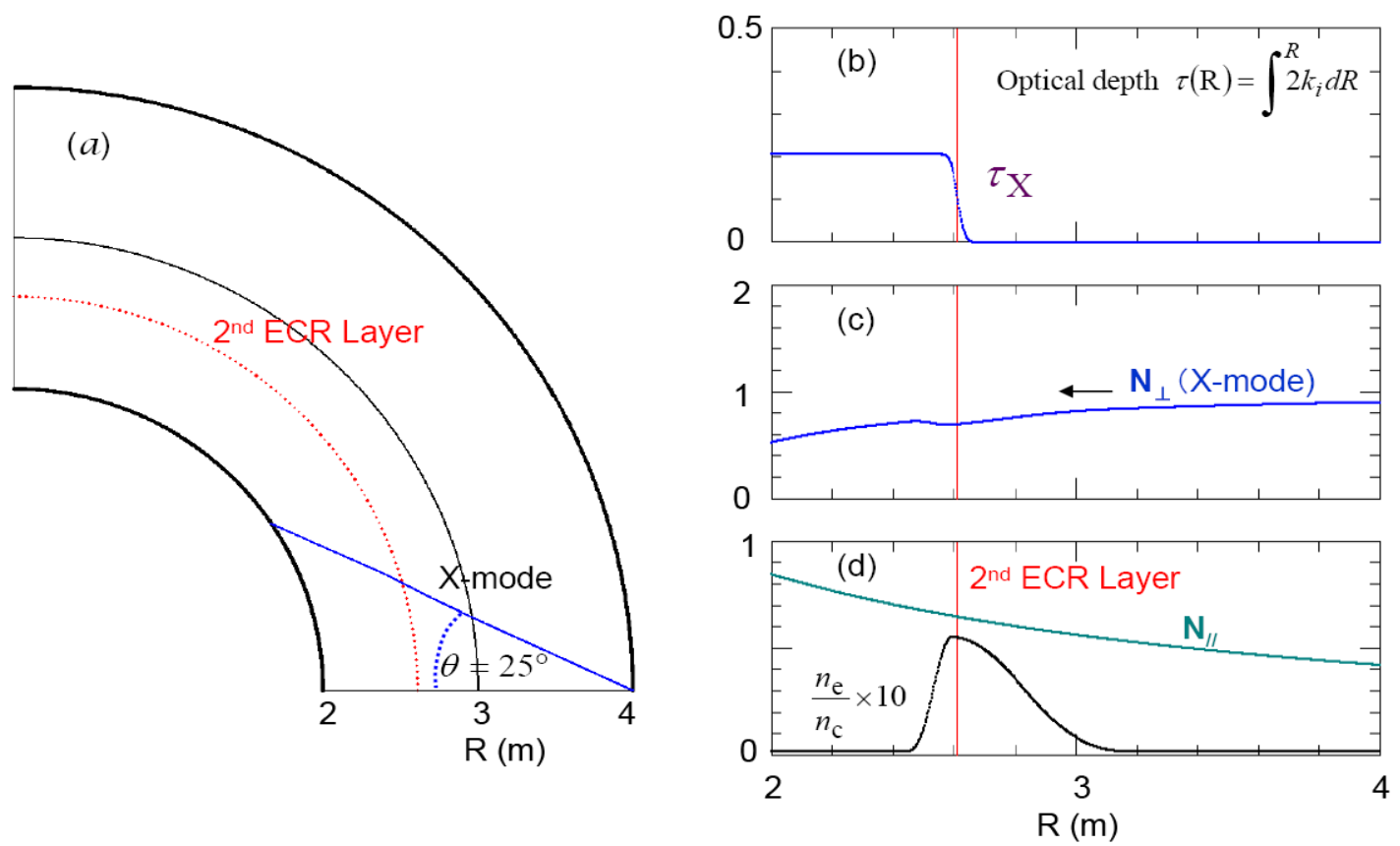

Figure 22. (a) Wave trajectory, (b) optical depth, (c) refractive index and (d) electron density profile for the case of second harmonic X-mode injection on the mid-plane. Electron temperature $(65 \mathrm{eV})$ is uniform. frequency $=110 \mathrm{GHz}$

Table 1. Optical depths of fundamental O-wave and reflected $\mathrm{X}$ wave ${ }^{*}$ denotes the values in figure 20)

\begin{tabular}{lllllll}
\hline$n_{e}\left(10^{19} \mathrm{~m}^{-3}\right)$ & 0.8 & 1.6 & 1.6 & 1.6 & 4.0 & $N_{\|}$ \\
$T_{e}(\mathrm{eV})$ & 65 & 65 & 300 & 600 & 120 & on inboard wall \\
\hline$\theta_{\text {inj }}=25$ degree & $0.034^{*}$ & 0.065 & 0.27 & 0.50 & 0.26 & 0.85 \\
Reflected X & $39^{*}$ & 22 & 95 & 190 & 17 & 0.85 \\
$\theta_{\text {inj }}=20$ degree & 0.048 & 0.09 & 0.40 & 0.73 & 0.37 & 0.68 \\
Reflected X & 25 & 13 & 60 & 120 & 11 & 0.68 \\
$\theta_{\text {inj }}=15$ degree & 0.057 & 0.12 & 0.49 & 0.94 & 0.26 & 0.52 \\
Reflected X & 13 & 6.5 & 31 & 61 & 6 & 0.52 \\
\hline
\end{tabular}

ECR layer from inboard, the ECR ellipsis appears on the backward wing in velocity space, where the waves might drive a backward current and are completely consumed before reaching the favorable wing for ECCD. Thus the wave access to the ECR layer from outboard is more advantageous than from inboard.

Tables 1 and 2 show optical depths for various conditions in the injection angle and plasma density and temperature for the fundamental O-wave injection and the second harmonic X-wave injection in figures 20 and 22, respectively. In large devices with strong toroidal fields, once a small closed flux surface is formed such as one in figure 17, 
Table 2. Optical depths of 2 nd harmonic $\mathrm{X}$-wave (* denotes the value in figure 22 )

\begin{tabular}{llllll}
\hline$n_{e}\left(10^{19} \mathrm{~m}^{-3}\right)$ & 0.8 & 1.6 & 1.6 & 1.6 & 4.0 \\
$T_{e}(\mathrm{eV})$ & 65 & 65 & 300 & 600 & 120 \\
\hline$\theta_{i n j}=28$ degree & 0.17 & 0.30 & 1.2 & 2.3 & waves not reach \\
$\theta_{i n j}=25$ degree & $0.20^{*}$ & 0.37 & 1.6 & 3.0 & 0.4 \\
$\theta_{i n j}=20$ degree & 0.24 & 0.47 & 2.0 & 3.8 & 1.5 \\
$\theta_{i n j}=15$ degree & 0.26 & 0.52 & 2.3 & 4.4 & 2.2 \\
\hline
\end{tabular}

both the electron density and temperature inside the flux surface may easily increases beyond the radiation barrier. Then the absorption of the second harmonic $\mathrm{X}$ wave improves drastically and the wave may well play the final role, that is, replacement of the equilibrium current to the ECCD current. On the other hand, in the case of fundamental $\mathrm{O}$ mode injection at an oblique angle, the reflected $\mathrm{X}$ wave is quite suitable to the first and second roles but is not quite useful to the final ECCD since the X wave approaches to the ECR layer from higher field side and absorption of primal $\mathrm{O}$ wave is not good.

In the experiments in the LATE device $[4,5,6]$ initial closed flux surfaces always appear near the fundamental ECR layer, after which the closed flux surface expands beyond the second harmonic resonance layer, generating a low aspect ratio configuration. This result seems to reflect the fact that in small devices absorption at second harmonic EC resonance is too weak to sustain bulk as well as CFP electrons. The situation may change in large devices.

\section{Discussions}

While the model can handle the case with non uniform $B_{V}$ field, we assumed uniform $B_{V}$ for simplicity. Actually it may be advantage in large devices to use $B_{V}$ field with an appropriate decay index to have a mirror vacuum field [12]. Figure 17 suggests that initial closed flux surface is so small that it is vital to steer and focus the EC waves onto this sweet spot to enlarge the flux surface. This requires good stability of vertical position of plasma cross section, which may be realized with a large decay index. Furthermore, initiation of discharge by EC waves may be more easy in a mirror field.

While present model can not handle the case in which $B_{V}$ field is temporally changed during development of flux surface from open to close, it may be useful to employ appropriate change of $B_{V}$ field, slow ramp-up of $B_{V}$ for example. When initial $B_{V}$ is lowered below 30 Gauss the energy range of initial CFP electrons is also lowered compared with the case in figure 18(a).

Finally note that present Fokker-Planck treatment of CFP electrons is a rough approximation. First, the CFP electrons rather continuously collide with bulk particles when they go trough the plasma while they receive a kick from the waves only when 
they traverse the thin ECR layer as appeared in figures 9 and 15. Second, the process of initiation of closed flux surface involves change of current generation mechanism. For accurate analyses more delicate treatment for time evolution of CFP electron velocity distribution may be needed. Nevertheless, simultaneous coincidence of the present model with two experiments in most small and large devices of LATE and JT-60U in terms of microwave power and driven current is remarkable, suggesting that the model takes into account the essence of initiation mechanism of closed flux surface by ECH.

\section{Summary}

A modeling for the non-inductive initiation of a closed flux surface observed in EC heated toroidal plasmas is presented. The model has been developed from an idea proposed in [5] that combined the previous works on two mechanisms of current generation, that is, preferential confinement of energetic electrons $[17,18]$ and bulk electron pressure driven equilibrium current $[23,24]$.

First, a pressure driven equilibrium toroidal current develops in open fields under a weak external vertical field so as to counter balance the pressure-ballooning and currenthoop forces of the plasma torus. The current is originated from the vertical charge separation drift of bulk thermal electrons in the toroidal filed. Both the fluid and the guiding-center-drift descriptions lead to the same pressure governing equation that calculates the equilibrium pressure profile for a given set of profiles of the current and external vertical field. A model function for the current is introduced and the pressure governing equation produces reasonable model sets of current and pressure profiles for the subsequent numerical analyses.

When the self field from the current develops and almost cancels out the external vertical field inside the current channel, a forward energetic part of electrons in the velocity space begin to make the CFP orbits. The CFP electrons are assumed to be generated by the EC heating of bulk electrons to the perpendicular direction and subsequent pitch angle scattering. They provide an additional current that closes the filed lines. Its current profile is obtained by summing up the contribution from every CFP electron orbit over the velocity distribution estimated using the Fokker Planck equation.

The model is examined for experiments both in the small low aspect ratio device of LATE and in the large conventional device of JT-60U with a search for appropriate modes of EC heating. Simultaneous coincidence of the model with these two experiments is obtained in terms of microwave power and driven current. The results predict that initiation of closed flux surface requires more and more EC power to generate the CFP electrons as the plasma major radius increases. Especially, careful injection of high $N_{\|}$ EC waves are required for large devices, both for initiation of a closed flux surface and for subsequent enlargement of the flux surface by usual ECCD onto the closed flux area. 


\section{Acknowledgment}

The present work was supported by the KAKENHI and NIFS collaboration program in Japan. Professor Yuji Nakamura kindly advised that the Fokker Planck equation was useful for estimation of CFP electrons distribution on the velocity space.

\section{Appendix A. Toroidal current profile}

The current profiles used in the profile are generated based on the following analytic form,

$$
\begin{aligned}
j_{\phi} & =j_{0}\left[1-\rho^{2}(x, y)\right]^{\alpha} & & \leq \leq \rho(x, y) \leq 0.7 \\
& =j_{0}\left[C_{1}(1-\rho(x, y))+C_{2}(1-\rho(x, y))^{2}\right] & & 0.7 \leq \rho(x, y) \leq 1 \\
& =0 & & 1 \leq \rho(x, y)
\end{aligned}
$$

where $j_{0}$ is the peak value and

$$
\rho(x, y)=\frac{\sqrt{\left(x+\frac{\delta}{\kappa^{2}} y^{2}\right)^{2}+\frac{1-\sigma^{2}}{\kappa^{2}} y^{2}}+\sigma\left(x+\frac{\delta}{\kappa^{2}} y^{2}\right)}{1-\sigma^{2}}
$$

and

$$
x=\frac{R-R_{P}}{a}, \quad y=\frac{Z}{a}, \quad \kappa=\frac{b}{a}, \quad \sigma=\frac{R_{P}-R_{0}}{a}<0
$$

with the minor radius $a$. Other parameters, $\alpha, \delta, \kappa$ and $\sigma$ are the peaking factor, triangularity, vertical elongation and radial shift of current peak, respectively, as shown in figure A1.

The multiply factors $C 1$ and $C 2$ in equation (A1) are adjusted so as that two different functions are continuously connected at $\rho(x, y)=0.7$. Equation (A2) suggests that $\rho$ represents "radius" and equation (A2) is rewritten as

$$
\left(x+\frac{\delta}{\kappa^{2}} y^{2}+\rho \sigma\right)^{2}+\frac{y^{2}}{\kappa^{2}}=\rho^{2}
$$

Substitution of $\rho=0$ into this equation gives the peak point of current density, $R=R_{P}$ and $Z=0$, and substitution of $\rho=1$ gives the current boundary.

In order to generate the self field numerically from the analytical current profile (equation(A1)) we first divide the poloidal cross section into fine square cells to make a grid mesh and distribute a large number of filament currents on every edge of square or cross point of mesh lines so that they match to the analytical profile. Second, the self filed from these filament currents is calculated using Biot-Savart law on every central point 


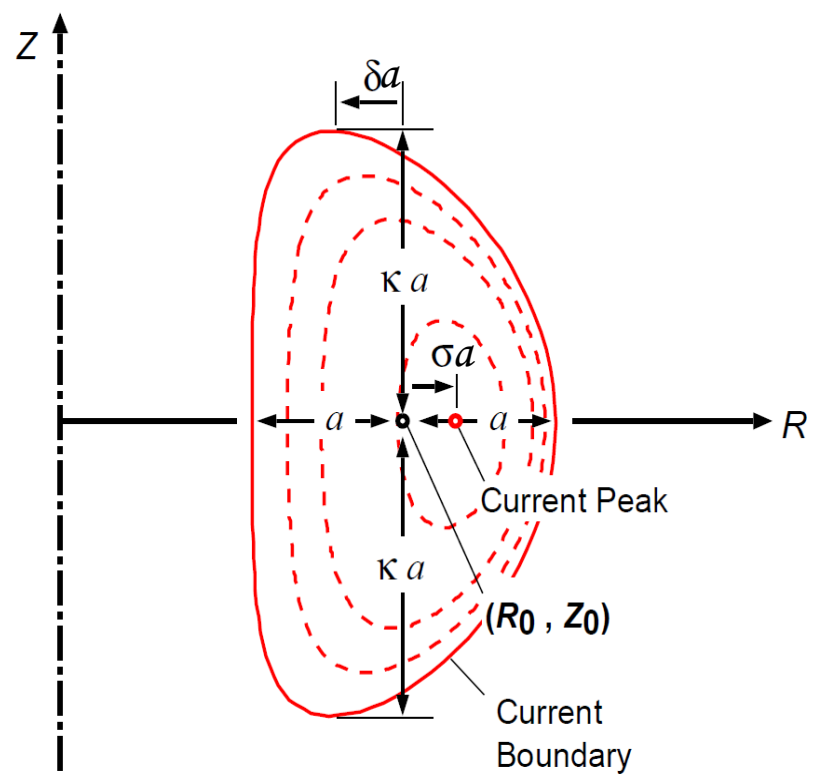

Figure A1. Current Profile Model

of the squares, making a dataset of the self-field on another grid mesh that shifts both vertically and horizontally from the current mesh by a half side of the square. Finally we numerically obtain the self field at any location using the spline interpolation for this dataset. Current profiles depicted in figures in the present paper are those reproduced from this numerical field profiles using Ampere's law.

\section{References}

[1] Kubo S. et al 1983 Phys. Rev. Lett. 501994

[2] Forest C. B. et al 1992 Phys. Rev. Lett. 683559

[3] Forest C. B. et al 1994 Phys. Plasmas 11568

[4] Maekawa T. et al 2005 Nucl. Fusion 451439

[5] Yoshinaga T. et al 2006 Phys. Rev. Lett. 96125005

[6] Yoshinaga T. et al 2007 Nucl. Fusion 47210

[7] Ejiri A. et al 2006 Nucl. Fusion 46709

[8] Meyer H. et al 2009 Nucl. Fusion 49104017

[9] Yoshinaga $T$. et al in Fusion Energy 2008 (Proc. 22nd Int. Conf. Geneva, 2008) (Vienna: IAEA) CD-ROM file EX/P6-9 and http://wwwnaweb.iaea.org/napc/physics/FEC/FEC2008/html/index.htm

[10] Tan Y. et al 2011 Nucl. Fusion 51063021

[11] Jackson G.L. et al 2011 Nucl. Fusion 51083015

[12] Uchida M. et al 2011 Nucl. Fusion 51063031

[13] Uchida M. et al 2010 Phys. Rev. Lett. 104065001

[14] Nishio S. et al 2004 Proc. 20th Int. Conf. on Fusion Energy 2004 (Vilamoura, Portugal, 2004) (Vienna: IAEA) CD-ROM file FT/P7-35 and http://wwwnaweb.iaea.org/napc/physics/fec/fec2004/datasets/index.html

[15] Tobita K. et al 2006 Fusion Engineering and Design 811151 
[16] Gribov Y. et al Progress in the ITER Physics Basis Chapter 8: Plasma operation and control 2007 Nucl. Fusion 47 S385

[17] Wong K.-L. et al 1980 Phys. Rev. Letters 45117

[18] Shimozuma T. et al 1985 J. Phys. Soc. Jpn. 541360

[19] Peng M. Y-K. and Borowski S.K. and Kamamsh T. 1978 Nucl. Fusion 181489

[20] Yoshikawa S. et al 1963 Phys. Fluids 61506

[21] Nishi S. et al 2010 Plasma Phys. Control. Fusion 52065011

[22] Nishi S. et al 2010 Plasma Phys. Control. Fusion 52125004

[23] Parail V. V. et al 1985 Proc. 10th Int. Conf. on Plasma Physics and Controlled Nuclear Fusion (London, 1984) vol 1 IAEA-CN-44/F-IV-4 (Vienna: IAEA) p 605

[24] Zakharov L. E. and Pereverzev G. V. 1988 Sov. J. Plasma Phys. 1475

[25] Goldston R. J. and Rutherford P. H., Introduction to Plasma Physics, (Institute of Physics Publishing, Bristol and Philadelphia, 1995) (see Fig.10.8)

[26] Shafranov V. D., in Review of Plasma Physics Review to Plasma Physics (Consultants Bureau, New York, 1966), Vol. 2.

[27] Hirshman S.P. and Neilson G.H. 1986 Phys. Fluids 29790

[28] Karney C.F.F. 1986 Comput. Phys. Rep. 4183

[29] Maekawa T. et al 2011 Plasma Science and Technology, 13342

[30] Maekawa T. et al 1980 J. Phys. Soc. Jpn. 48, 247

[31] Igami H. et al 2004 Plasma Phys. Control. Fusion 46 261-275 (see Appendix A)

[32] Maehara T. et al 1998 Nuclear Fusion 3839 (see Appendix A) 